\title{
The Rayleigh-Plesset equation: a simple and powerful tool to understand various aspects of cavitation.
}

\author{
Jean-Pierre FRANC \\ University of Grenoble, France
}

\begin{abstract}
This chapter is a general introduction to cavitation. Various features of cavitating flows are analyzed on the basis of the Rayleigh-Plesset equation. They concern not only the simple configuration of a single spherical bubble but also complex cavitating flows as those observed in cavitating turbopumps. Scaling rules, erosive potential, thermodynamic effect, supercavitation, traveling bubble cavitation, cavitation modeling are some of the topics addressed here. They are examined through this simple, basic equation which proves to be a quite useful tool for a first approach of real cavitation problems.
\end{abstract}

\section{Introduction}

Cavitation is the development of vapor structures in an originally liquid flow. Contrary to boiling, the phase change takes place at almost constant temperature and is due to a local drop in pressure generated by the flow itself.

The occurrence of low pressure regions in flows is a well-known phenomenon. For example, in the case of a Venturi, i.e. a converging duct followed by a diverging one, the velocity is maximum at the throat where the cross section is minimum. Then, according to Bernoulli equation, the pressure is minimum there and the risk of cavitation is maximum.

Another example is the flow around a foil at a given angle of attack which is representative of that around the blades of a hydraulic machine. From classical hydrodynamics, it is well-known that the foil is subject to a lift because of a lower pressure on the suction side in comparison to the pressure side. Hence, the suction side is expected to be the place where cavitation will first develop.

A final example is that of vortices which are very common structures in many flows. Because of the rotation and the associated centrifugal forces, the pressure in the core of such structures is lower than outside. Hence vortices are likely to cavitate in their core. There are actually many situations in which cavitating vortices can be observed as tip vortices or coherent vortical structures in turbulent flows like wakes or shear layers.

As known from basic thermodynamics, phase change from liquid to vapor occurs at the vapor pressure $p_{v}$ which depends only upon the temperature. It is usually a good approximation to consider that the critical pressure for the onset of cavitation is the vapor pressure $p_{v}$, although some deviations discussed later may occur. 


\subsection{The cavitation number}

The degree of development of cavitation is characterized by a non dimensional parameter, the cavitation number $\sigma$, defined by:

$$
\sigma=\frac{p_{r e f}-p_{v}}{\frac{1}{2} \rho V^{2}}
$$

In this expression, $p_{\text {ref }}$ is a reference pressure taken at a given point in the liquid flow and $V$ is a characteristic flow velocity. Both parameters need to be precisely specified for each practical situation. As an example, in the case of a cavitating flow past a single foil in a hydrodynamic tunnel (see e.g. Figure 3), the reference pressure and velocity are usually chosen as the pressure and velocity in the undisturbed liquid flow, far from the foil.

A non cavitating flow corresponds to large values of the cavitation number. This is easy to understand since large values of the cavitation number usually correspond to large values of the reference pressure. Then, it can be expected that the pressure will be everywhere above the vapor pressure and the flow will remain free of cavitation. It is clear that the cavitation number has no influence on the fully wetted flow which will remain the same whatever the cavitation number may be, provided it is large enough for the flow to remain actually non cavitating. This number is a pertinent parameter only for cavitating flows for which it can be considered as a scaling parameter which measures the global extent of cavitation.

The onset of cavitation generally appears for a critical value of the cavitation number known as the incipient cavitation number $\sigma_{i}$. Starting from the fully wetted flow, cavitation inception can be reached either by decreasing the reference pressure or increasing the flow velocity, both leading to a decrease in cavitation number. Any further decrease will lead to an additional development of cavitation. In the case of Figure 3 for instance, the cavity will grow and its length will increase with a decrease in cavitation number leading to a longer cavity comparable to the supercavity shown in Figure 5. If the reference pressure is now increased, it is generally observed that cavitation disappears for a critical cavitation number somewhat higher than $\sigma_{i}$. Incipient and desinent cavitation numbers are often different and an hysteresis effect is often observed.

\subsection{Main types of cavitation}

Looking at real cavitating flows as that in a cavitating turbopump (Figure 1) or around a propeller (Figure 2), it appears that the liquid vapor interfaces have generally complicated shapes. There is a wide variety of types of cavitation and basically we can identify the following ones:

$\checkmark$ attached cavities as that shown in Figures 3 to 5. Cavitation appears here in the form of a cavity attached to the suction side of the foil. The type of cavitation shown in Figure 3 is known as partial cavitation since the cavity covers only partially the upper side. On the contrary, a supercavity as shown in Figure 5 fully covers the suction side and closes downstream the foil trailing edge.

$\checkmark \quad$ traveling bubble cavitation with more or less isolated bubbles according mainly to the nuclei density in the free stream (Figures 6 to 8).

$\checkmark$ cavitation clouds which can take various forms. Figure 9 gives an example of two clouds shed by an unsteady partial cavity. This is an illustration of the partial cavitation instability 
which is triggered by a re-entrant jet developing upward from the closure region of the cavity.

$\checkmark$ cavitating vortices which can be more or less structured. They are observed in particular at the tip of three-dimensional foils (Figure 10) or in the turbulent wake of bluff bodies where they are less organized because of turbulence (Figure 11).

Secondary effects as interactions between bubbles or with solid walls, fission, coalescence, interface instabilities, re-entrant jet, turbulence... can dramatically complicate previous basic shapes of liquid / vapor interfaces at both large and small scales. The analysis of cavitation can then be particularly difficult because of the geometric complexity of the liquid / vapor interfaces.

\subsection{Overview of chapter}

Despite this complexity, many basic results can be rather easily derived from the RayleighPlesset equation. This equation, presented in section 2, applies to an isolated spherical bubble which is assumed to remain spherical all along its life. It gives how its radius changes because of the change in pressure it might go through during its life. This is the case, for instance, of an initial microbubble or cavitation nucleus carried by a liquid flow which undergoes pressure changes as it goes along the blades of a hydraulic machine. It grows in low pressure regions, becomes a macroscopic cavitation bubble and finally collapses downstream where the pressure recovers.

Section 3 is devoted to the presentation of a few basic results on single bubbles. First equilibrium is considered and it is shown that the critical pressure for the explosive growth of a nucleus may be significantly smaller than vapor pressure because of surface tension. The two main stages in the typical evolution of a cavitation bubble, i.e. growth and collapse, are then addressed with emphasis on the collapse time, which is a characteristic time scale of great importance in cavitation. Finally, it is shown that a bubble in a liquid is an oscillator because of the elastic behavior of the non condensable gas generally enclosed; the period of oscillation, which is another characteristic time scale, is introduced.

Section 4 is devoted to the presentation of non dimensional forms of the Rayleigh-Plesset equation from which a few conclusions on cavitation scaling are deduced. A first form based on the introduction of characteristic times - pressure, viscous and surface tension times - allows the estimation of the relative importance of each of these phenomena on the dynamics of a single bubble. A second form appropriate to the case of a bubble traveling on the suction side of a blade allows the derivation of scaling laws for traveling bubble cavitation.

Section 5 addresses thermal effects in cavitation. An extended form of the Rayleigh-Plesset equation including thermal effects is derived. Once more it is made non dimensional in order to develop a practical criterion for the estimation of the so called thermodynamic effect in cavitation.

Section 6 is relative to supercavitation, a field in which the Rayleigh-Plesset equation is surprisingly applicable. According to the Logvinovich independence principle, the dynamics of any cross section of a supercavity is independent of the neighboring ones and can be modeled by a Rayleigh type equation. This section shows that the Rayleigh equation, originally derived for spherical bubbles, may also be useful for other cavities whose geometry is actually far from being spherical. 
Section 7 is devoted to an analysis of cavitation erosion using once more the Rayleigh equation. Firstly, it is shown that the spherical collapse of a bubble generates a pressure pulse of high amplitude that can largely exceed the yield strength of usual materials and hence cause damage. The flow aggressiveness of a single bubble and consequently of a whole cavitating flow is then analyzed with a special emphasis on the influence of velocity on erosive potential. The section closes with a few general remarks on the erosive potential of various cavitating flows, still based on a discussion of the Rayleigh equation.

In section 8 , it is shown that the dynamics of other types of cavities, as ring bubbles, can be modeled by a Rayleigh-Plesset type equation, with some changes and additional terms which take into account the specificities of such cavitating structures, as vorticity.

The chapter ends with a brief presentation of a cavitation model based on the RayleighPlesset equation and often used for simulation. The liquid is assumed to carry cavitation nuclei and the Rayleigh-Plesset equation, which models the evolution of individual bubbles in the cluster, is coupled to Navier-Stokes equations. Such a technique is appropriate to the modeling of complex real cavitating flows, as for instance cloud cavitation generated by a pulsating leading edge cavity. 


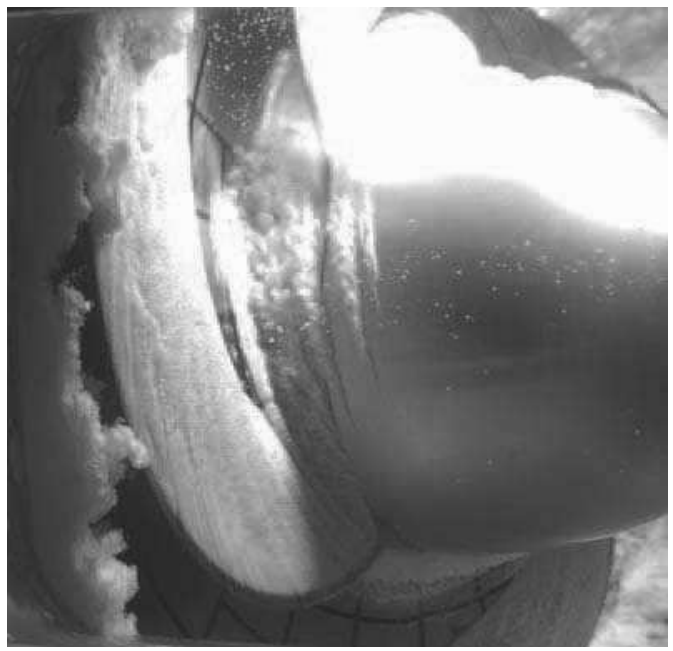

Figure 1. Cavitating flow in the inducer of a rocket engine turbopump (Courtesy of SNECMA Moteurs and CNES)

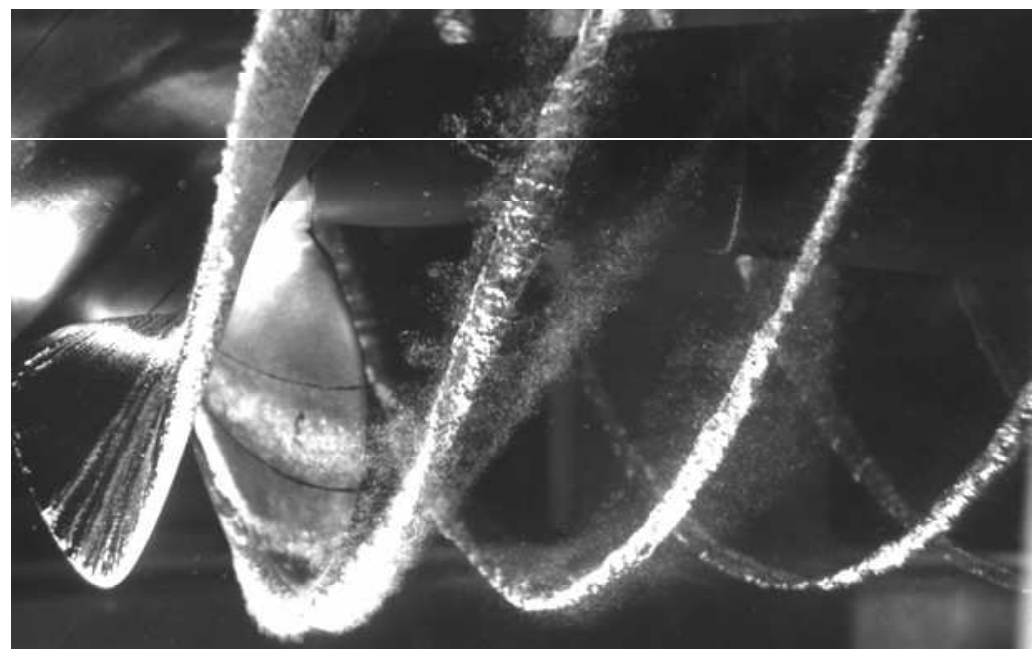

Figure 2. Cavitating flow in a marine propeller (Courtesy of DGA/BEC) 


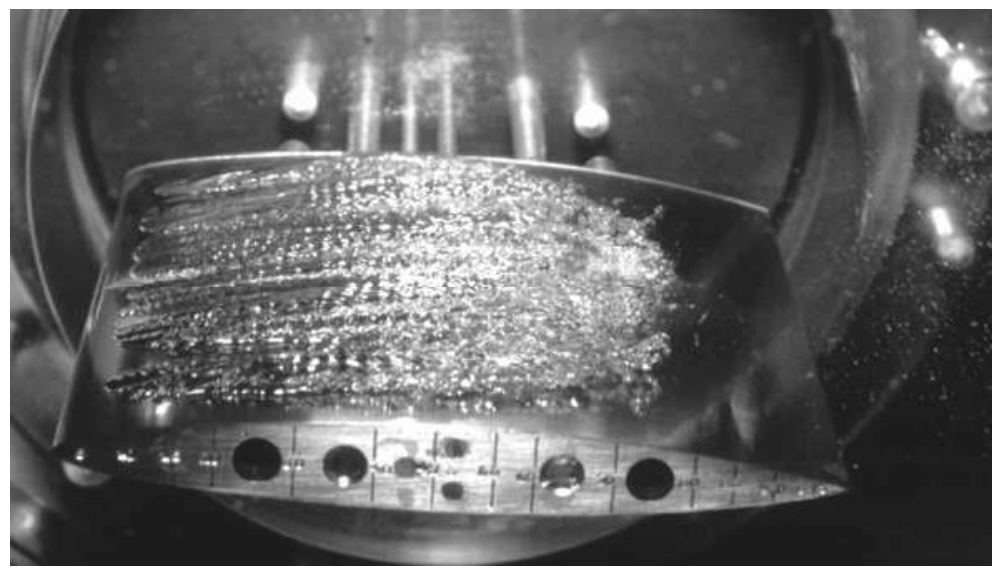

Figure 3. Partial cavity flow on a hydrofoil

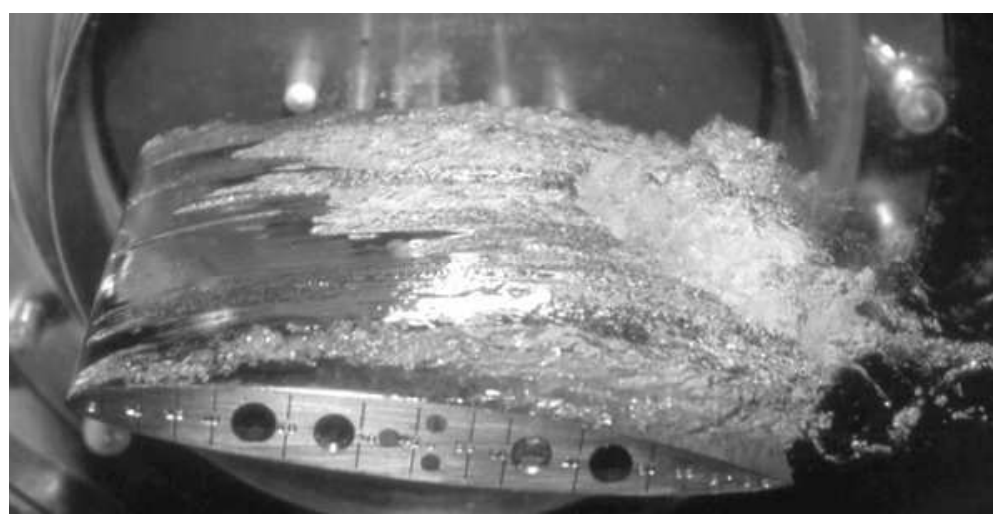

Figure 4. Unstable partial cavitation on a hydrofoil

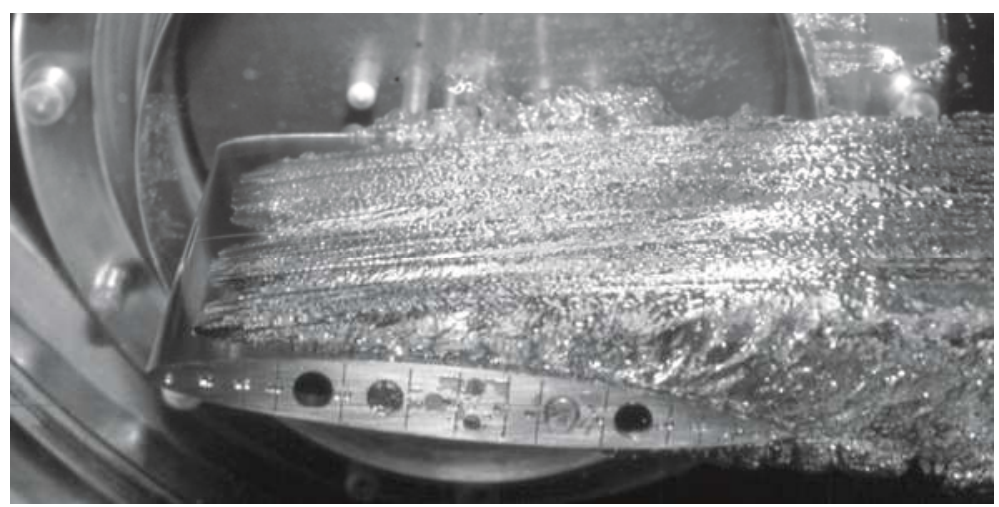

Figure 5. Supercavity flow around a hydrofoil in a cavitation tunnel 


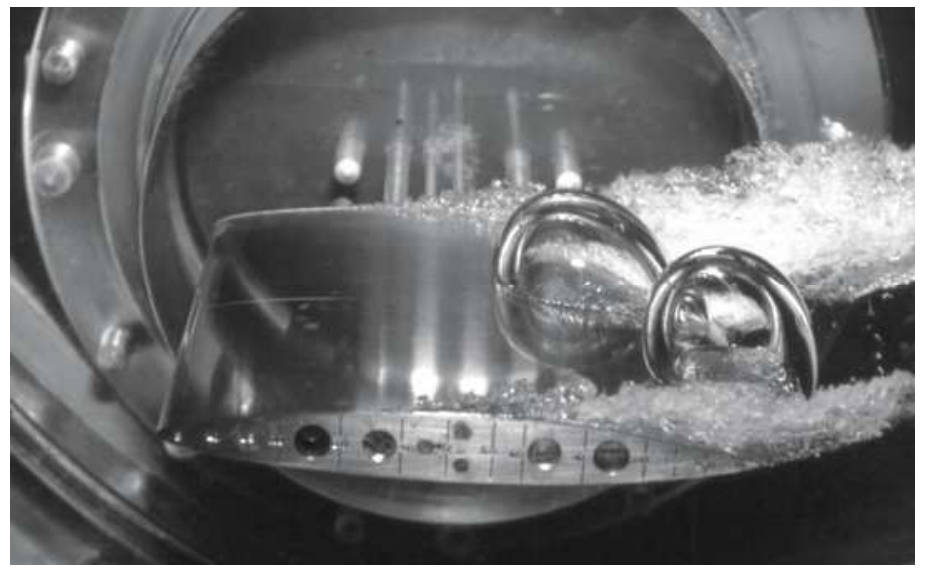

Figure 6. Two cavitation bubbles on the suction side of a hydrofoil

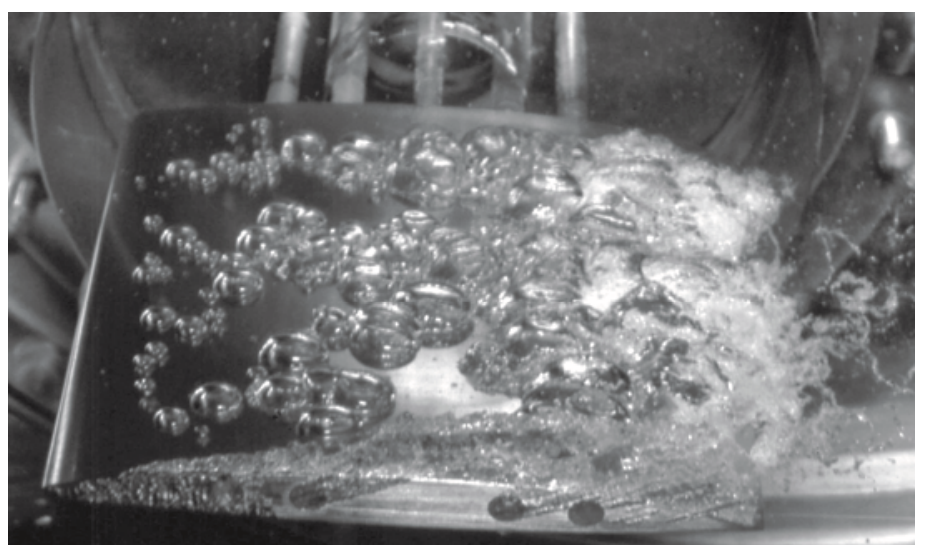

Figure 7. Traveling bubble cavitation at medium angle of attack

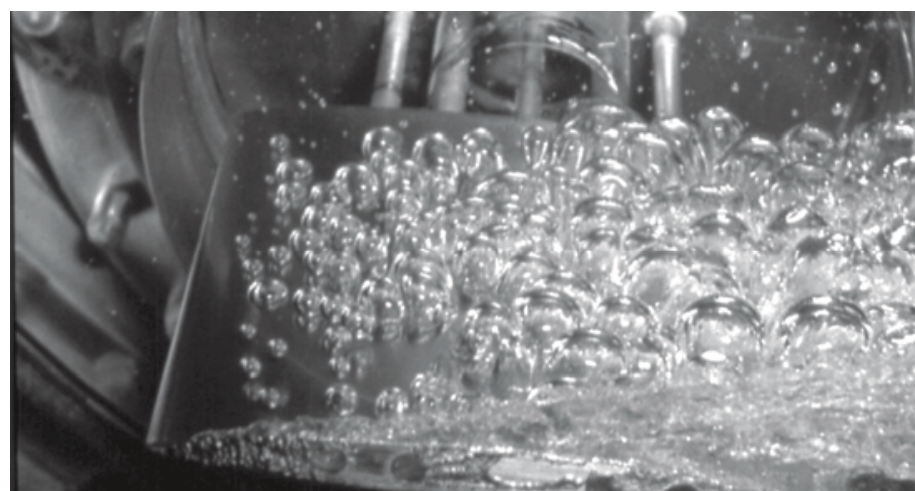

Figure 8. Traveling bubble cavitation at large angle of attack 


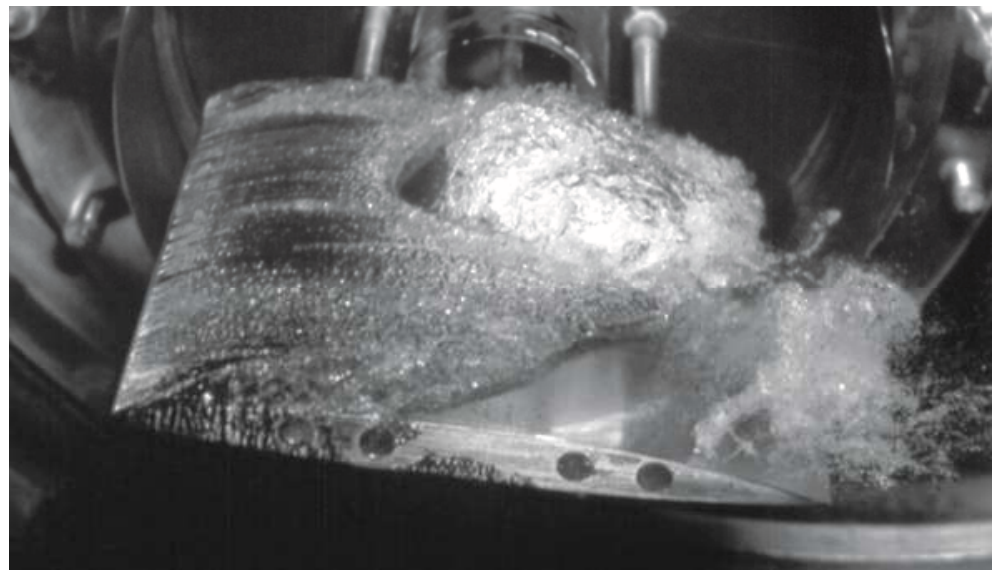

Figure 9. Unsteady leading edge cavity shedding cavitation clouds

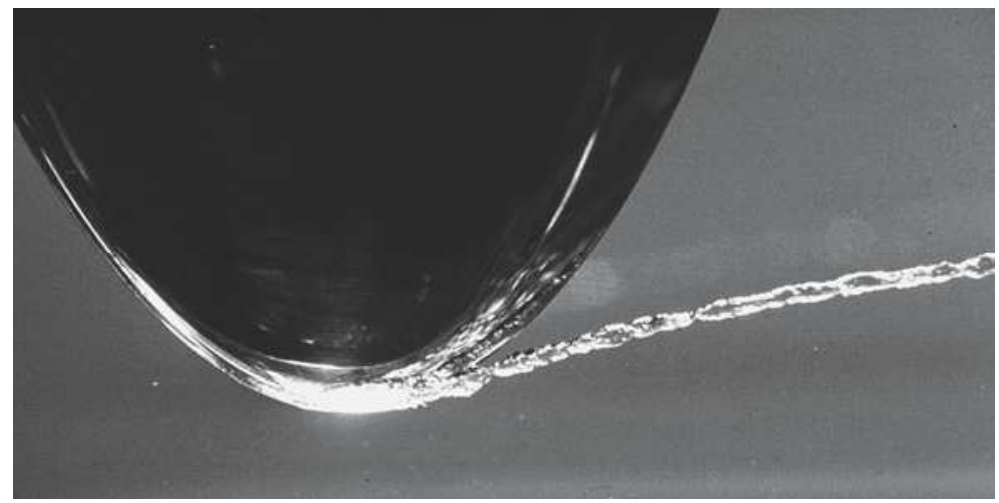

Figure 10. Vortex cavitation at the tip of a three dimensional foil

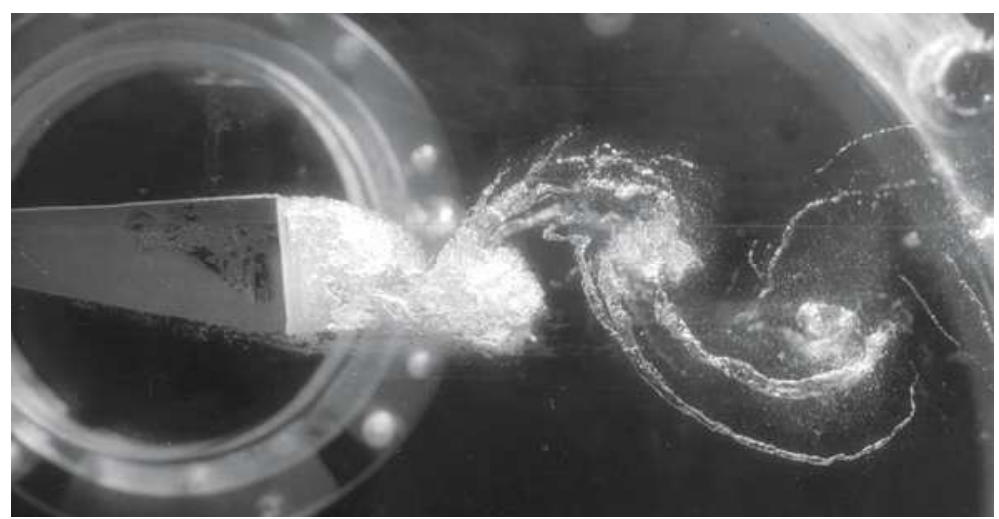

Figure 11. Cavitating vortices in the turbulent wake of a bluff body 


\section{The Rayleigh-Plesset equation}

\subsection{Cavitation nuclei}

Cavitation is generally initiated from microscopic nuclei carried by the flow. Such nuclei are points of weakness for the liquid from which macroscopic cavities are generated and grow in low pressure regions.

The simplest and most widely used model of nucleus is that of a microbubble. Such a microbubble, typically of a few microns in diameter, is assumed to be spherical and to contain a gaseous mixture made of the vapor of the liquid and possibly of non condensable gas. The presence of non condensable gas is quite general in practice. In the most common case of water, it is well-known that ordinary water contains dissolved air (essentially oxygen and nitrogen) at least if no special degassing procedure is applied to it. The presence of non condensable gas inside the bubbles is then due to the migration of gas by molecular diffusion through the bubble interface.

Figure 12 illustrates how a microbubble can give birth to a macroscopic cavitation bubble when moving along the suction side of a foil.

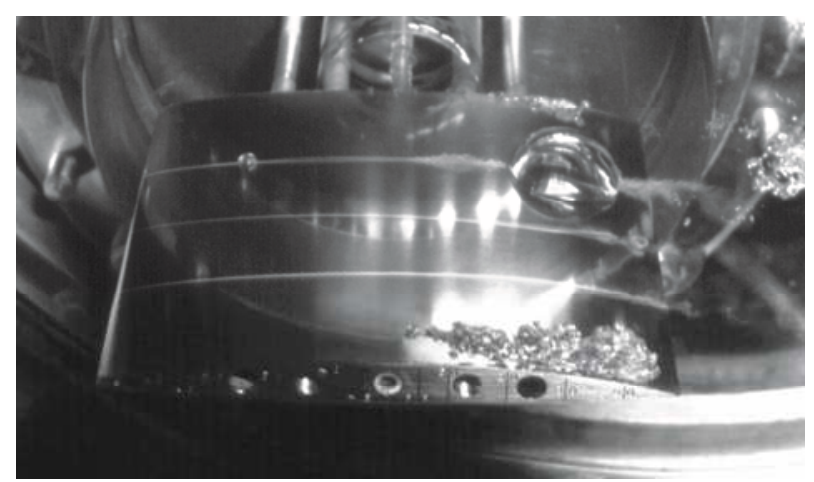

Figure 12. Growth of a nucleus on the suction side of a hydrofoil

\subsection{The dynamics of a spherical bubble}

It is then of major importance for the understanding of cavitation to be able to understand and predict the evolution of such a bubble. This is possible from the Rayleigh-Plesset equation. The driving parameter for the bubble dynamics is obviously the pressure. In the case of Figure 12 for example, the pressure which is applied to the bubble changes during its movement along the suction side. It is usually assumed to be the pressure of the original fully wetted flow at each successive location of the bubble center. This time dependent pressure that the bubble experiences is the driving factor of the bubble dynamics.

For simplicity, the bubble is assumed to evolve in a infinite medium at rest at infinity. The basic input for bubble dynamics is the instantaneous pressure law $p_{\infty}(t)$ applied to it. Any other 
flow than the pure radial one induced by the growth or collapse of the bubble is ignored. All the information on the original flow is then supposed to be included in the only $p_{\infty}(t)$ law.

The expected output is the time evolution of the bubble radius $R(t)$. It satisfies the following second-order differential equation known as the Rayleigh-Plesset equation:

$$
\rho\left[R \ddot{R}+\frac{3}{2} \dot{R}^{2}\right]=\left[p_{v}-p_{\infty}(t)\right]+p_{g 0}\left(\frac{R_{0}}{R}\right)^{3 k}-\frac{2 S}{R}-4 \mu \frac{\dot{R}}{R}
$$

The derivation of this equation together with the main required assumptions can be found in any textbook on cavitation (see e.g. Brennen (1995), Franc and Michel (2004)). In this equation, $\dot{R}$ and $\ddot{R}$ are the first and second order derivatives of the bubble radius with respect to time and $R_{0}$ is the initial bubble radius.

On the right hand side, four different terms appear. The first one $p_{v}-p_{\infty}(t)$, which measures the closeness of the applied pressure to the vapor pressure, is the driving term for the bubble evolution. It is the most fundamental one since the evolution of the bubble (growth, collapse, oscillations...) will depend essentially upon it.

The second one is the contribution of non condensable gas. Its derivation is based on several assumptions. First it is assumed that the mass of non condensable gas inside the bubble remains constant during its evolution. This is a simplifying assumption that could be evaluated by solving the mass diffusion equation, which is however far beyond the scope of the present introductory chapter.

Secondly, this constant mass of gas is assumed to follow a polytropic thermodynamic behavior characterized by a given polytropic coefficient $k$. If the behavior is isothermal, $k=1$. If it is adiabatic, $k$ is the ratio $\gamma$ of the heat capacities of the enclosed gas. To resolve the ambiguity, it would be necessary to solve an energy equation, which is not essential at this step. The gas transformation can often be assumed as isothermal since the characteristic time for the evolution of a nucleus in real cavitating flows is usually much larger than that required for heat transfer so that temperature equilibrium is continuously achieved. However, for big bubbles resulting of the explosion of a nucleus, the behavior tends to be adiabatic (cf. Franc and Michel (2004)). Nevertheless, we will keep both possibilities by introducing a polytropic coefficient $\mathrm{k}$.

The polytropic behavior is described by the following law between the partial pressure $p_{g}$ of the gas inside the bubble and its radius $R$ :

$$
p_{g} R^{3 k}=p_{g 0} R_{0}^{3 k}
$$

where subscript 0 refers to initial conditions. In this equation, $R^{3}$ is actually representative of the bubble volume. From the previous equation, the second term on the right hand side of the Rayleigh-Plesset equation appears to be simply the instantaneous partial pressure $p_{g}$ of the gas inside the bubble.

The third term is the contribution of surface tension. $S$ is the usual surface tension coefficient expressed in $\mathrm{N} / \mathrm{m}$ or $\mathrm{J} / \mathrm{m}^{2}$. Since $R$ appears at the denominator, this term is important only for small radii. 
The last term accounts for the effect of dynamic viscosity $\mu$ of the liquid. Dissipation due to viscosity appears to be proportional to bubble deformation rate $\dot{R}$ and inversely proportional to bubble radius so that it is expected to be significant only for small radii as surface tension.

When the effects of non condensable gas, surface tension and viscosity are negligible, as it is the case for big enough bubbles, the Rayleigh-Plesset equation reduces to the simple Rayleigh equation :

$$
\rho\left[R \ddot{R}+\frac{3}{2} \dot{R}^{2}\right]=p_{v}-p_{\infty}(t)
$$

Furthermore, if the applied pressure $p_{\infty}$ is constant, the Rayleigh equation can be integrated once to give the bubble interface velocity:

$$
\dot{R}^{2}=\frac{2}{3} \frac{p_{v}-p_{\infty}}{\rho}\left[1-\left(\frac{R_{0}}{R}\right)^{3}\right]
$$

\section{A few basic results}

\subsection{Bubble equilibrium}

Although it is not necessary to know the Rayleigh-Plesset equation to study the equilibrium of a bubble, the condition for equilibrium can easily be deduced from the Rayleigh-Plesset equation by setting all time derivatives to zero and assuming constant the external pressure $p_{\infty}$. For the analysis of equilibrium, it is common to assume the gas transformation as isothermal $(k=1)$ since the temperature can be considered as continuously fixed by that of the liquid. If so, we get the following equilibrium condition:

$$
p_{\infty}=p_{g 0}\left[\frac{R_{0}}{R}\right]^{3}+p_{v}-\frac{2 S}{R}
$$

This equation expresses that the difference between the pressure inside and outside the bubble is due to surface tension. By solving it with respect to R, we can obtain the radius of equilibrium of a bubble at any external pressure $p_{\infty}$. The corresponding relationship between pressure at infinity and equilibrium radius is shown in Figure 13.

It is important to observe that the equilibrium is not always stable. This point is connected to the existence of a minimum for the equilibrium curve. The corresponding pressure and radius, called critical (for reasons which will become clear at the end of this section) and labeled here by subscript $c$, are given by:

$$
\left\{\begin{array}{l}
R_{c}=\sqrt{\frac{3 p_{g 0} R_{0}{ }^{3}}{2 S}} \\
p_{c}=p_{v}-\frac{4 S}{3 R_{c}}
\end{array}\right.
$$


Critical radius and pressure depend on surface tension $\mathrm{S}$ and on the group of parameters $p_{g 0} R_{0}{ }^{3}$. Since $R_{0}{ }^{3}$ is proportional to the volume of the bubble, $p_{g 0} R_{0}{ }^{3}$ is actually proportional to the mass of non condensable gas in the bubble. As a nucleus is completely defined by the quantity of non condensable gas it contains (which is assumed constant), it can also be characterized by either its critical radius or, what is most common, by its critical pressure.

To come back to the problem of stability, consider three different nuclei in equilibrium under conditions 1, 2 and 3 as shown in Figure 13 and, in order to analyze the stability, let us assume that the pressure is, for example, slightly lowered. The equilibrium condition 3.1 is obviously no longer satisfied and the unbalance due to a lower pressure $p_{\infty}$ is such that the right hand side of the Rayleigh-Plesset equation becomes positive. Hence the bubble will grow as it can be reasonably expected following a pressure drop.

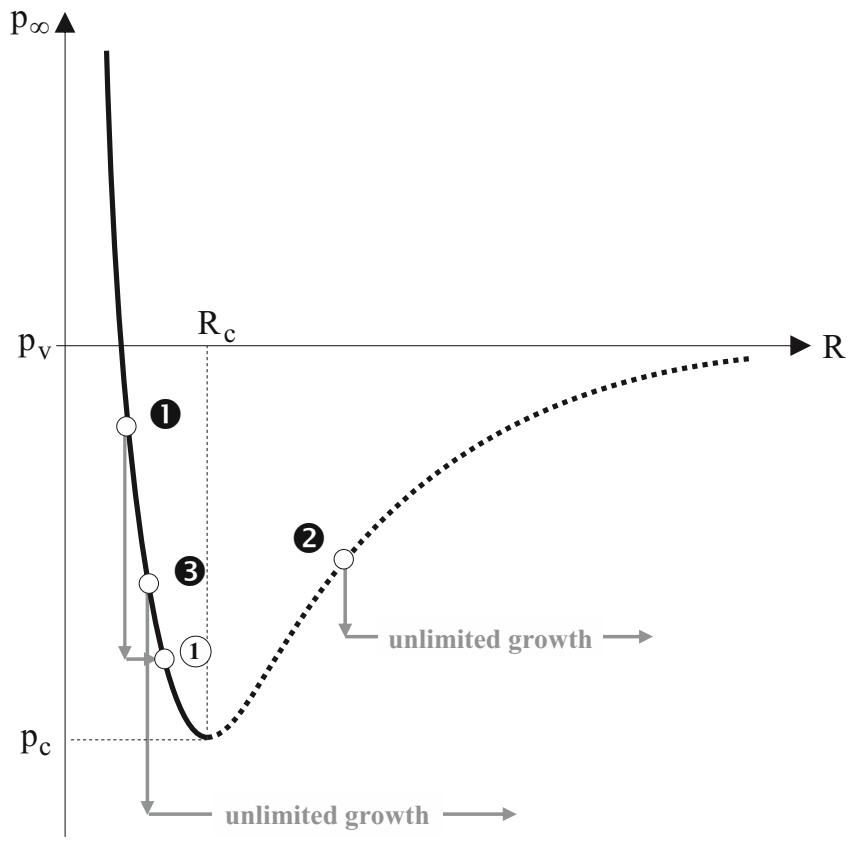

Figure 13. Equilibrium radius of a cavitation nucleus versus pressure at infinity

In the case of nucleus 1, it is clear that the bubble will reach a new equilibrium since the representative point of the bubble follows a path which crosses the equilibrium curve at a new point 1 '. This descending part of the equilibrium curve is then stable.

On the contrary, for nucleus 2 , the bubble will indefinitely grow without crossing the equilibrium curve, so that the equilibrium is unstable.

Finally, in situation 3 for which the pressure is lowered below the critical pressure which corresponds to the minimum of the equilibrium curve, the bubble will grow indefinitely as well without reaching a new equilibrium. Hence the critical pressure can actually be considered as a threshold for the microbubble to explode and become a macroscopic cavitation bubble. 
According to equation 3.2, the critical pressure is smaller than the vapor pressure and the difference is due to surface tension. It is negligible for large nuclei but can become important for small ones. As an example, a nucleus of radius $R_{0}=3 \mu \mathrm{m}$ in equilibrium in water $\left(p_{v}=2300 \mathrm{~Pa}, \mathrm{~S}=0.072 \mathrm{~N} / \mathrm{m}\right)$ at atmospheric pressure $p_{\infty}=10^{5} \mathrm{~Pa}$ will have a partial pressure of air inside equal to $p_{g 0}=p_{\infty}-p_{v}+2 S / R \cong 146000 \mathrm{~Pa}$, a critical radius $R_{c} \cong 9 \mu m$ and a critical pressure $p_{c}=p_{v}-10600 \mathrm{~Pa}$ which appears to be significantly smaller than the vapor pressure and even negative. A tension is then necessary to activate this nucleus and make it grow indefinitely.

The difference $p_{v}-p_{c}$ is known as the nucleus static delay. In the introduction of this chapter, we mentioned that the threshold pressure for cavitation is usually considered as the vapor pressure. The present model shows that the threshold pressure is actually the nucleus critical pressure which is smaller than the vapor pressure.

In ordinary water, there are generally many nuclei with a wide range of diameters. The weakest points are the biggest nuclei since their critical pressure is the largest. They will then cavitate first. The critical pressure of the biggest nuclei is known as the susceptibility pressure of the liquid sample. It is the critical pressure for cavitation. If no special treatment is applied to the liquid by removing big nuclei, the susceptibility pressure is expected to remain close to the vapor pressure and the assumption of a threshold for cavitation equal to the vapor pressure is quite appropriate.

\subsection{Bubble growth}

As already observed, the effects of non condensable gas, surface tension and viscosity become negligible when the cavitation bubble is much bigger than the original nucleus. If so, the simplified Rayleigh equation is applicable. If the applied pressure $p_{\infty}$ equals the vapor pressure $p_{v}$, the bubble is in equilibrium. If $p_{\infty}<p_{v}$, the bubble will grow $\left(R>R_{0}\right)$ and, according to Equation 2.4, the asymptotic growth rate for large radii is:

$$
\dot{R} \cong \sqrt{\frac{2}{3} \frac{p_{v}-p_{\infty}}{\rho}}
$$

This equation is valid quite early since, as soon as the cavitation bubble is three times bigger than the initial nucleus, the error on the estimation of the interface velocity using Equation 3.3 instead of Equation 2.4 is smaller than 2\%. Previous equation is important in cavitation as it gives the order of magnitude of the growth rate of a cavitation bubble when submitted to a given pressure $p_{\infty}<p_{v}$. Let us observe that simple dimensional arguments would have lead to the same formula, except for the numerical coefficient $\sqrt{2 / 3}$ which cannot be predicted from a pure dimensional analysis.

\subsection{Collapse of a pure vapor bubble}

If the applied pressure is higher than the vapor pressure, the bubble radius decreases $\left(R<R_{0}\right)$. This is the collapse phase. If we continue to ignore the effects of viscosity, non condensable gas and surface tension, the interface velocity during collapse is given by: 


$$
\dot{R} \cong-\sqrt{\frac{2}{3} \frac{p_{\infty}-p_{v}}{\rho}\left[\left(\frac{R_{0}}{R}\right)^{3}-1\right]}
$$

Integration of Equation 3.4 allows the computation of the collapse time or bubble lifetime i.e. the time necessary for the bubble to completely disappear until $\mathrm{R}$ vanishes $(R=0)$. This time is called the Rayleigh time and is given by:

$$
\tau_{p} \cong 0.915 R_{0} \sqrt{\frac{\rho}{p_{\infty}-p_{v}}}
$$

It is interesting to observe that, once more, a simple dimensional analysis would have lead to the same formula except for the coefficient 0.915 which could not be guessed. The subscript $\mathrm{p}$ just recalls that this characteristic time scale is directly connected to the pressure difference $p_{\infty}-p_{v}$ (see also section 4.1).

It is of crucial importance in cavitation to observe that during the collapse phase of a pure vapor bubble without non condensable gas inside as assumed here, the interface velocity $\dot{R}$ is always increasing and becomes infinite at the very end of the collapse $(R=0)$. This final behavior is of course not realistic providing evidence that some original assumptions are no longer physically valid at the ultimate stage of collapse. This is the case when assuming the liquid incompressible, which is obviously unacceptable when the interface velocity comes near to the speed of sound. This is also the case when ignoring the effect of non condensable gas since they are continuously compressed and their pressure drastically increases during collapse. This is another limiting factor which contributes to a reduction in the interface velocity during the final stage of collapse and possibly an inversion of the movement causing bubble rebound.

\subsection{Bubble resonance frequency}

A bubble in a liquid is a possible oscillator because of the elastic behavior of the non condensable gas that the bubble contains and the inertia of the liquid. Then, a natural resonance frequency is associated to any cavitation bubble in a liquid. However, dissipative losses as those due to viscosity or heat conduction for instance tend to damp out bubble oscillations. From the Rayleigh-Plesset equation, it is easy to predict the pulsating behavior of a bubble and in particular to compute its resonance frequency.

Consider a bubble of radius $R_{0}$ in equilibrium at pressure $p_{\infty 0}$. The partial pressure $p_{g 0}$ of non condensable gas in the bubble is given by the equilibrium condition (cf. Equation 3.1):

$$
p_{\infty 0}=p_{v}+p_{g 0}-\frac{2 S}{R_{0}}
$$

Suppose that the pressure $p_{\infty}$ oscillates around equilibrium $p_{\infty 0}$ with pulsation $\omega$ and a small amplitude $\delta p$ according to the relation $p_{\infty}=p_{\infty 0}+\delta p \sin \omega t$. The variations in bubble radius can be computed using the Rayleigh-Plesset equation which can be written as follows after making use of the equilibrium condition: 


$$
\rho\left[R \ddot{R}+\frac{3}{2} \dot{R}^{2}\right]=-\delta p \sin \omega t+p_{g 0}\left[\left(\frac{R_{0}}{R}\right)^{3 k}-1\right]+2 S\left(\frac{1}{R_{0}}-\frac{1}{R}\right)-4 \mu \frac{\dot{R}}{R}
$$

By introducing the displacement $\delta R=R-R_{0}$ instead of $R$ and assuming that $\delta R$ remains small which is normally the case if the driving pressure fluctuation $\delta p$ is small enough, the previous equation can be linearized as follows:

$$
\rho R_{0} \ddot{\delta R}+4 \mu \frac{\dot{\delta R}}{R_{0}}+\left(3 k p_{g 0}-\frac{2 S}{R_{0}}\right) \frac{\delta R}{R_{0}} \cong-\delta p \sin \omega t
$$

This is the usual equation of a forced harmonic oscillator whose natural frequency is:

$$
f_{0}=\frac{1}{2 \pi R_{0}} \sqrt{\frac{1}{\rho}\left[3 k p_{g 0}-\frac{2 S}{R_{0}}\right]}
$$

Making use of the equilibrium condition 3.6 to estimate the partial pressure of non condensable gas, the resonance frequency is more straightly given by the following equation:

$$
f_{0}=\frac{1}{2 \pi R_{0}} \sqrt{\frac{1}{\rho}\left[3 k\left(p_{\infty 0}-p_{v}+\frac{2 S}{R_{0}}\right)-\frac{2 S}{R_{0}}\right]}
$$

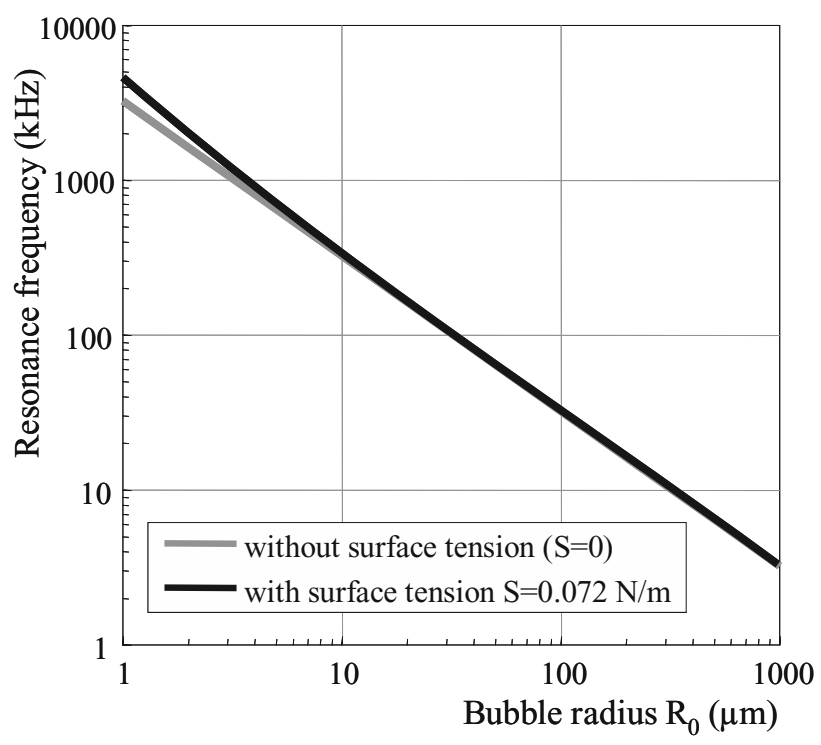

Figure 14. Resonance frequency for cavitation bubbles in water oscillating adiabatically at atmospheric pressure $\left(p_{\infty 0}=10^{5} \mathrm{~Pa}, p_{v}=2300 \mathrm{~Pa}, k=\gamma=1.4, S=0.072 \mathrm{~N} / \mathrm{m}, \rho=1000 \mathrm{~kg} / \mathrm{m}^{3}\right)$.

Without surface tension $(S=0)$, the frequency is inversely proportional to bubble radius.

The resonance frequency of cavitation bubbles of various radii in water oscillating adiabatically $(k=\gamma=1.4)$ at atmospheric pressure is shown in Figure 14. The influence of surface 
tension appears negligible except for small radii. The resonance frequency can then be considered as inversely proportional to the bubble radius.

The period of natural oscillation is another important characteristic time for a cavitation bubble. In the present case of a bubble submitted to an oscillating pressure field, the amplitude of the harmonic response of the bubble depends greatly on the ratio of the frequency of the applied pressure to the bubble resonance frequency, as for any forced oscillator. More generally, the period of natural oscillations has to be compared to the characteristic time of pressure variations. If they are of the same order of magnitude, bubble oscillations are expected.

Furthermore, let us observe that the second term on the left hand side of Equation 3.8, which involves the first derivative of the bubble radius, demonstrates the damping effect of viscosity on bubble oscillations.

\section{Cavitation scaling}

\subsection{Characteristic time scales for an isolated pure vapor bubble}

The Rayleigh-Plesset equation is a rather convenient basis to point out characteristic time scales of the cavitation phenomenon. In the previous sections, we already introduced two of them. Firstly, the Rayleigh time, which depends upon the bubble initial radius and the difference between the applied pressure and the vapor pressure. It is typical of the bubble lifetime when collapsing. Secondly, the resonance frequency, which is mainly connected to the elastic behavior of the non condensable gas, and which characterizes the period of natural oscillations. In the present section, two new time scales are introduced. They are connected to two other physical phenomena, namely viscosity and surface tension. By comparing these various characteristic time scales, it is possible to estimate the importance of each associated physical phenomenon on the dynamics of a cavitation bubble.

We consider here a pure vapor bubble without any non condensable gas inside and we suppose it is submitted to a varying pressure at infinity $p_{\infty}(t)$. Let $a$ be a characteristic length scale for the bubble radius. If we are interested in the first stage of the bubble evolution, it is natural to choose the initial radius $R_{0}$ for $a$. From purely dimensional arguments, we can then define a viscous time $\tau_{v}$ and a surface tension time $\tau_{S}$ on the basis of length scale $a$ and either viscosity $v$ or surface tension $S$. It is easy to check that the two following variables are actually measured in time units:

$$
\left\{\begin{array}{l}
\tau_{v}=\frac{\rho a^{2}}{4 \mu} \\
\tau_{S}=a \sqrt{\frac{\rho a}{2 S}}
\end{array}\right.
$$

In addition, we consider a pressure time defined by: 


$$
\tau_{p}^{\prime}=a \sqrt{\frac{\rho}{p_{\infty \text { ref }}-p_{v}}}
$$

In this equation, $p_{\infty \text { ref }}$ is a reference pressure which is supposed to be a suitable order of magnitude for the applied pressure $p_{\infty}(t)$. This time scale is similar to the Rayleigh time $\tau_{p}$ introduced in section 3.3 devoted to the analysis of bubble collapse, except that the constant 0.915 has been ignored and the initial bubble radius $R_{0}$ has been replaced by $a$, to be more general.

The discussion on the relative importance of these various time scales is conducted on the basis of a non dimensional form of the Rayleigh-Plesset equation (2.1). The bubble radius is made non dimensional using length scale $a$ :

$$
\bar{R}=\frac{R}{a}
$$

As for the characteristic time scale, it is a priori unknown. We will then introduce a new constant $\tau$ which is assumed to be a characteristic time for the bubble evolution. One of the conclusions of the present approach will be the actual estimation of this time scale. A non dimensional time $\bar{t}$ is then defined by:

$$
\bar{t}=\frac{t}{\tau}
$$

It is of major importance to carefully choose the reference length scale $a$ and time scale $\tau$. Actually, they must be pertinent orders of magnitude of the bubble radius and evolution time. In other words, the corresponding non dimensional variables $\bar{R}$ and $\bar{t}$ must be of the order of unity. At the beginning of bubble evolution, the initial radius is obviously a suitable length scale as already mentioned. However, since the bubble size may change by several orders of magnitude with time, it may be necessary to adapt the characteristic length scale during bubble evolution. Furthermore, if the bubble evolution is sufficiently regular, such a well thought non dimensional procedure ensures that the derivatives $\dot{R}$ and $\bar{R}$ are also of the order of unity, as usually assumed in classical dimensional analysis.

With this change of variables, the Rayleigh-Plesset equation takes the following non dimensional form (the effect of non condensable gas is not included here):

$$
\bar{R} \ddot{\bar{R}}+\frac{3}{2} \dot{\bar{R}}^{2}=-\left(\frac{\tau}{\tau_{p}^{\prime}}\right)^{2} \frac{p_{\infty}(t)-p_{v}}{p_{\infty \mathrm{ref}}-p_{v}}-\left(\frac{\tau}{\tau_{S}}\right)^{2} \frac{1}{\bar{R}}-\frac{\tau}{\tau_{v}} \frac{\dot{\bar{R}}}{\bar{R}}
$$

From previous equation, it is easy to estimate the relative importance of the three terms driven respectively by pressure, surface tension and viscosity, which appear on the right hand side. Since all variables including the non dimensional radius $\bar{R}$ and its derivatives are of the order of unity, as mentioned above, the dominating term is the one whose characteristic time is the smallest. The two others can then be neglected. This procedure allows the identification of the physical mechanism which controls the evolution of the bubble.

To illustrate the approach, the characteristic pressure, surface tension and viscous times are plotted in Figure 15 against the bubble radius $a$. The pressure time depends upon the reference pressure $p_{\infty \text { ref }}$ which has been chosen equal to 1 bar in Figure 15 as an example. From this fig- 
ure, it appears that the pressure controls the dynamics of the cavitation bubble in a wide range of radii. Surface tension becomes dominating for bubbles smaller than typically $1 \mu \mathrm{m}$ in radius whereas viscous effects are predominant only for very small bubbles, smaller than about $0.1 \mu \mathrm{m}$.

In addition, such an approach can give the order of magnitude of the characteristic time $\tau$ for the evolution of the bubble, which remains unknown so far. As an example, consider the case of the collapse of a vapor bubble under a constant pressure $p_{\infty}(t)=p_{\infty \text { ref }}$. If the collapse is pressure dominated, the two last terms in Equation (4.5) are negligible. Since the left hand side is of the order of unity (still because of the suitable choice in reference length and time scales), it is concluded that time $\tau$ must be of the same order of magnitude that pressure time $\tau_{p}^{\prime}$. This shows that the collapse time is actually given by the pressure time as already concluded in section 3.3 from a detailed computation of the bubble lifetime. Similarly, considering the case of a collapse dominated by surface tension, it can be concluded that the surface tension time $\tau_{S}$ is a relevant order of magnitude for the collapse time under the only effect of surface tension.

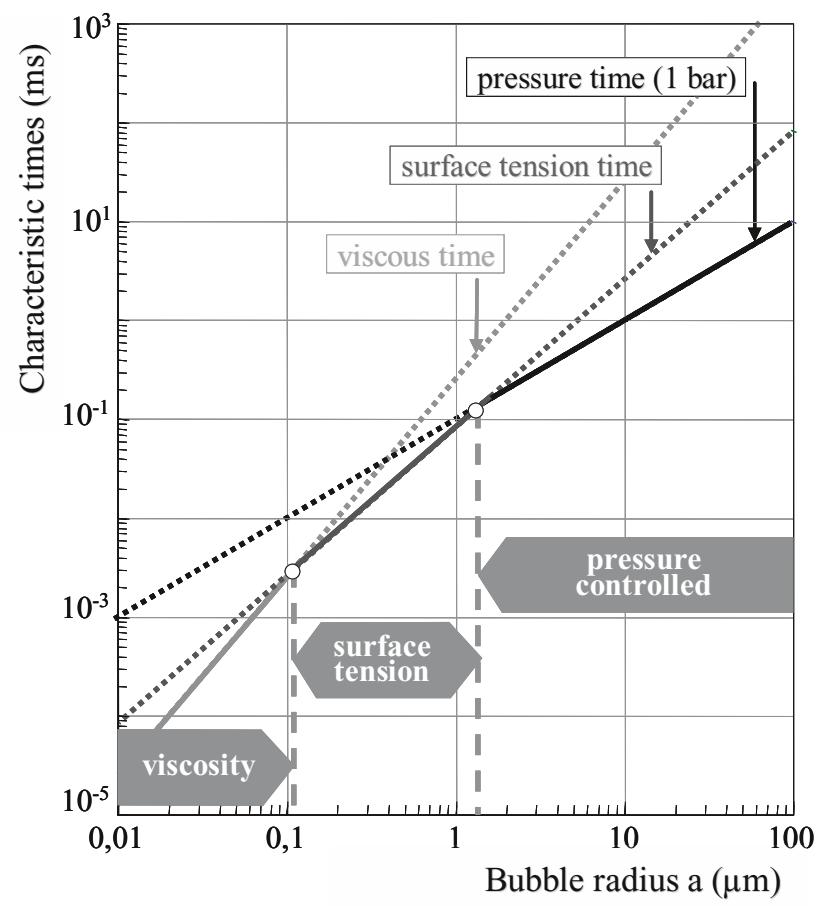

Figure 15. Characteristic time scales versus bubble radius. Pressure time is estimated at atmospheric pressure $\left(p_{\infty \text { ref }}=1\right.$ bar $)$.

\subsection{Scaling law for traveling bubble cavitation}

We consider here the case of traveling bubble cavitation as shown in Figures 6 to 8. In such a cavitating flow, the cavitation bubbles are generated from nuclei carried by the oncoming liquid. They grow on the suction side of the foil before collapsing in the pressure recovery region. 
The problem which is addressed here is the influence of foil size on the dynamics of cavitation bubbles. In particular, we will discuss the scaling law to be satisfied to ensure similarity between a prototype at full scale and a model at smaller scale.

This problem can be approached by using the Rayleigh equation and computing the evolution of a bubble moving along the suction side. The pressure law $p_{\infty}(t)$ which has to be introduced in the Rayleigh equation is deduced from the computation of the fully-wetted flow around the foil. It is assumed to be given by the successive pressures that the bubble center encounters as it moves along the foil. Clearly, this method ignores possible interactions between bubbles and also the change in the original fully-wetted flow pressure distribution due to the development of cavitation.

In addition, the effects of surface tension, viscosity and gas content are neglected, which is valid as soon as the microbubble becomes a macroscopic cavitation bubble. It is then quite good to consider the Rayleigh equation (2.3):

$$
R \frac{d^{2} R}{d t^{2}}+\frac{3}{2}\left(\frac{d R}{d t}\right)^{2}=\frac{p_{v}-p_{\infty}(t)}{\rho}
$$

In this problem, the time variable $t$ is not really a relevant parameter and it is more appropriate to consider the distance $x$ along the foil. Time derivatives are then changed into space derivatives using the mean flow velocity $V$ of the bubble according to the common transformation $x=V t$. The previous equation is then transformed as follows:

$$
R \frac{d^{2} R}{d x^{2}}+\frac{3}{2}\left(\frac{d R}{d x}\right)^{2}=\frac{p_{v}-p_{\infty}(x)}{\rho V^{2}}
$$

where $p_{\infty}(x)$ is the pressure to which the bubble is submitted at any station $x$ along the foil.

The approach is still based upon a non dimensional form of the Rayleigh equation. The difference in comparison with previous section 4.1 is that the bubble radius $R$ and the distance $x$ are made non dimensional using here the chord length $c$, so that the new non dimensional variables are:

$$
\left\{\begin{array}{l}
\bar{R}=\frac{R}{c} \\
\bar{x}=\frac{x}{c}
\end{array}\right.
$$

In addition, we introduce the usual non dimensional pressure coefficient $C_{p}$ defined by:

$$
C_{p}(x)=\frac{p_{\infty}(x)-p_{r e f}}{\frac{1}{2} \rho V^{2}}
$$

where $p_{\text {ref }}$ is a reference pressure already introduced in section 1.1. Using definition 1.1 of the cavitation parameter, equation (4.7) takes the following non dimensional form:

$$
\bar{R} \frac{d^{2} \bar{R}}{d \bar{x}^{2}}+\frac{3}{2}\left(\frac{d \bar{R}}{d \bar{x}}\right)^{2}=-\frac{1}{2}\left(C_{p}+\sigma\right)
$$


Let us observe that, in the present approach, the bubble is assumed to have a constant velocity $V$. If, at any time, the bubble is supposed to take the local flow velocity on the foil, which is more realistic, an additional term appears in equation (4.10) (see e.g. Franc \& Michel (2004)). The general conclusions remain however unchanged.

Consider two similar flows around geometrically similar foils at exactly the same angle of attack, the only difference being the chord length which is respectively $c$ and $\lambda c$. The pressure coefficient distribution is then exactly the same. Provided the cavitation number is the same for both flows, the non dimensional solution $\bar{R}(\bar{x})$ of equation (4.10) is the same too. In other words, at the same relative position $\bar{x}$ on the foil, the radius of the cavitation bubble is proportional to the chord length $c$. The bigger the foil, the bigger the bubble, as schematically shown in Figure 16.

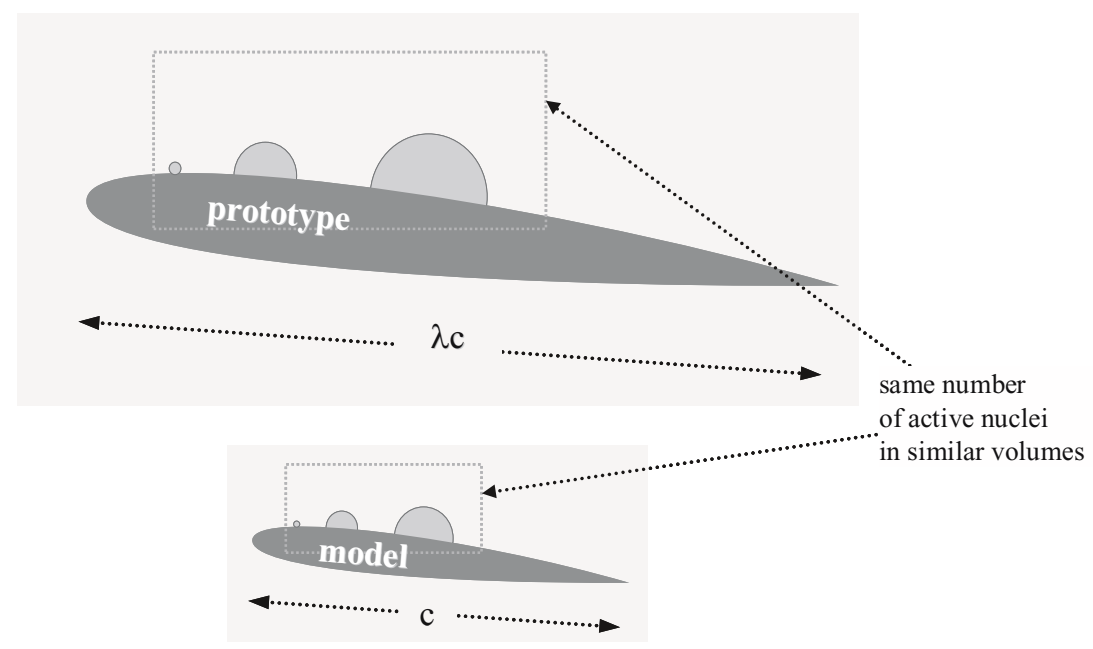

Figure 16. Scaling rules between model and prototype for traveling bubble cavitation

If we now consider the case of traveling bubble cavitation with several bubbles growing together on the suction side, both cavitating flows, model and prototype, will be similar if, in addition, similar volumes of liquid contain exactly the same number of active nuclei. If so, there will be statistically the same number of cavitation bubbles on the foils. The cavitation pattern and then the hydrodynamic performance of the foils will be statistically identical. In terms of nuclei density i.e. of number of microbubbles per unit volume, this requires that the nuclei density $N_{c}$ for model tests be $\lambda^{3}$ times larger than the nuclei density $N_{\lambda c}$ for the prototype. The following scaling law must then be satisfied between model and prototype:

$$
\frac{N_{c}}{N_{\lambda c}}=\lambda^{3}
$$

This $\lambda^{3}$ law suggests that the density of active nuclei must be much larger for the model at small scale than for the prototype at full scale.

This scaling law does not need to be satisfied when the foil suction side is saturated with cavitation bubbles for large nuclei densities. When saturation occurs, the foil is fully covered with 
bubbles which merge and form a kind of continuous cavity where the pressure is set to the vapor pressure. Beyond saturation, foil performance becomes independent of nuclei content and there is no need to satisfy the previous $\lambda^{3}$ scaling law. It is the reason why model tests are sometimes conducted with maximum nuclei seeding to get rid of this constraint and also be sure not to overestimate cavitation performances.

\section{Thermodynamic effect}

As mentioned in the introduction of this chapter, it is generally assumed that temperature is uniform and equal to the liquid bulk temperature all over a cavitating flow. This is the case for water at normal temperature. Strictly speaking, the temperature variation induced by cavitation is so small in cold water that it can actually be neglected.

However, this is not the case for all fluids. For cryogenic liquids used in rocket propulsion for instance, the temperature in the cavitating region $T_{c}$ may be significantly lower than that of the liquid bulk $T_{\infty}$. The physical mechanism for this drop in temperature is the following.

Cavitation is basically a vaporization of the liquid which involves a latent heat as for any phase change. Since the cavities are growing inside the liquid itself, the latent heat of vaporization can only be supplied by the liquid surrounding the cavities. Hence, the liquid close to the two phase region, and consequently this region itself, is cooled down.

Although the phenomenon is reversed in the collapse region, the growth region is generally prevailing. As an example, consider the case of a partial cavity attached to the leading edge of a foil, as shown in figure 17. Vaporization takes place on a large and steady upstream part of the cavity whereas condensation occurs around cavity closure, where vapor structures are shed and entrained by the liquid flow. The temperature drop is maximum close to the cavity leading edge and the temperature progressively increases along the cavity and reaches the liquid bulk temperature around cavity closure. There is no overshoot in mean temperature connected to condensation at closure mainly because of an important dispersion of vapor structures and a high turbulence level which both contribute to make the temperature practically uniform in this zone. The following approach will then be focused on the case of a growing bubble which is representative of most real situations.

In the absence of thermodynamic effect, the pressure inside the bubble would be $p_{v}\left(T_{\infty}\right)$. Because of the drop in temperature, the actual pressure is somewhat smaller. It is equal to $p_{v}\left(T_{c}\right)$, the vapor pressure at the actual bubble temperature $T_{c}$. Note that it is quite a general result to assume thermodynamic equilibrium in cavitating flows. It is only in very special situations, as during the ultimate stage of collapse for instance, that this condition might not be satisfied. The difference in pressure $p_{v}-p_{\infty}$ responsible for bubble growth is then smaller and bubble growth is slowed down. Therefore, the thermodynamic effect tends to reduce the development of cavitation. This is a general conclusion valid for any type of cavitation. The length of leading edge cavities for instance may be significantly reduced by thermodynamic effect. 


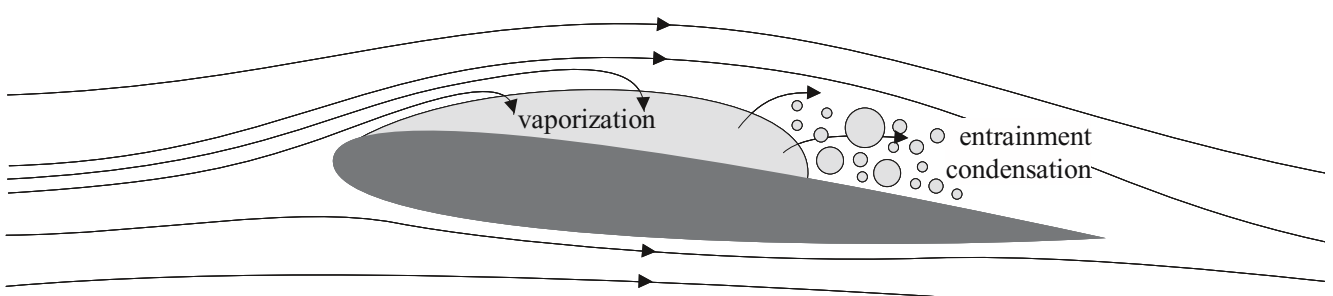

Figure 17. Schematic view of leading edge cavitation

In practice, the problem is to evaluate the order of magnitude of the temperature drop as a function of the thermodynamic properties of the liquid and its vapor and also of the cavitating flow conditions. This requires to write an energy balance which has been ignored so far.

Consider a cavitation nucleus which is growing in a liquid medium whose temperature far from the bubble is $T_{\infty}$. Let $R(t)$ be the bubble radius at time $t$ and $T_{c}(t)$ the temperature inside. Initially, it is assumed that the radius of the original microbubble is negligible and that the internal temperature is that of the liquid $T_{\infty}$.

During bubble growth, the heat necessary for vaporization is supposed to be supplied to the interface by conduction through the liquid. Hence, a thermal boundary layer develops on the bubble wall. The liquid temperature drops from $T_{\infty}$ to $T_{c}$ through this boundary layer (see figure 18). As for any diffusive process, the order of magnitude of the boundary layer thickness is $\sqrt{\alpha_{\ell} t}$ where $\alpha_{\ell}=\lambda_{\ell} /\left(\rho_{\ell} c_{p \ell}\right)$ is the thermal diffusivity of the liquid $\left(\lambda_{\ell}, \rho_{\ell}\right.$ and $c_{p \ell}$ are the conductivity, density and heat capacity of the liquid). The typical temperature gradient within the boundary layer is $\Delta T / \sqrt{\alpha_{\ell} t}$ where $\Delta T=T_{\infty}-T_{c}$. According to Fourier's law, the conductive heat flux towards the interface is then of the order of $\lambda_{\ell} \Delta T / \sqrt{\alpha_{\ell} t}$.

The energy balance expresses that, at any time, the heat supplied by conduction to the interface of area $4 \pi R^{2}$ is used for vaporization and causes the increase of the mass of vapor $\frac{4}{3} \pi R^{3} \rho_{v}$ inside the bubble ( $\rho_{v}$ is the vapor density). Hence, the energy balance writes:

$$
\lambda_{\ell} \frac{\Delta T}{\sqrt{\alpha_{\ell} t}} 4 \pi R^{2}=\frac{d}{d t}\left(\frac{4}{3} \pi R^{3}\right) \rho_{v} L
$$

where $L$ is the latent heat of vaporization.

Previous equation leads to the following estimate of the temperature drop at any time:

$$
\Delta T \cong \frac{\dot{R} \sqrt{t}}{\sqrt{\alpha_{\ell}}} \frac{\rho_{v} L}{\rho_{\ell} c_{p \ell}}
$$

Let us observe that the quantity

$$
\Delta T^{*}=\frac{\rho_{v} L}{\rho_{\ell} c_{p \ell}}
$$

has temperature units. This group of parameters appears commonly when thermodynamic effect is concerned. Experiments show that $\Delta T^{*}$ is a relevant order of magnitude of the temperature drop due to thermal effects. Figure 19 gives some typical values of $\Delta T^{*}$ for different fluids at different temperatures. From this figure, the thermodynamic effect appears negligible for water at 
room temperature whereas it becomes significant for hot water. It is important for liquid hydrogen around $20 \mathrm{~K}$ as used in rocket engines.

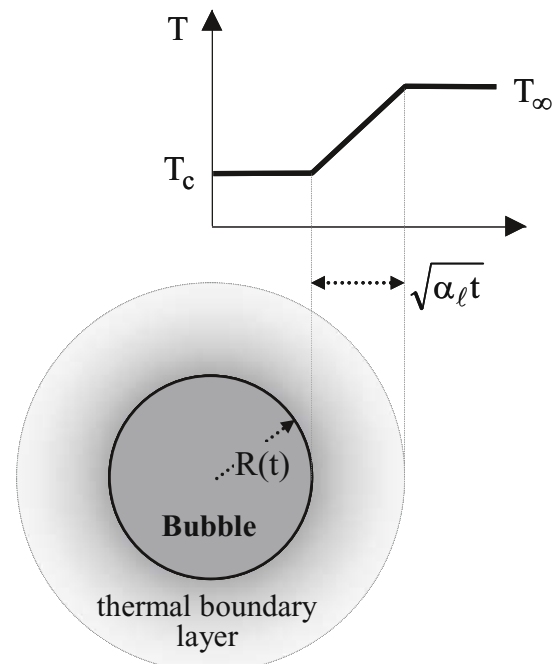

Figure 18. The thermal boundary layer on the bubble interface

The $B$ factor of Stepanov is the non dimensional temperature drop defined by:

$$
B=\frac{\Delta T}{\Delta T^{*}}
$$

This parameter has a simple physical interpretation. Consider that a volume $v_{v}$ of vapor is produced and that the heat required for vaporization is taken from a volume $\mathcal{V}_{\ell}$ of liquid which is supposed to be cooled of $\Delta T=T_{\infty}-T_{c}$. If so, the heat balance writes:

$$
\rho_{v} V_{v} L=\rho_{\ell} V_{\ell} c_{p \ell} \Delta T
$$

Hence, the $B$ factor appears to be the ratio $\mathcal{V}_{v} / \mathcal{V}_{\ell}$ of the volume of vapor produced to the volume of liquid that supplies the latent heat of vaporization.

The drop in temperature $\Delta T$ is accompanied by a drop in vapor pressure $\Delta p_{v}$ given by:

$$
\Delta p_{v}=p_{v}\left(T_{\infty}\right)-p_{v}\left(T_{c}\right) \cong \frac{d p_{v}}{d T} \Delta T
$$

The slope of the vapor pressure curve $d p_{v} / d T$ can be estimated using the famous thermodynamic equation of Clapeyron in which we can usually assume that vapor density is negligible with respect to liquid density:

$$
L=T\left[\frac{1}{\rho_{v}}-\frac{1}{\rho_{\ell}}\right] \frac{d p_{v}}{d T} \cong \frac{T}{\rho_{v}} \frac{d p_{v}}{d T}
$$




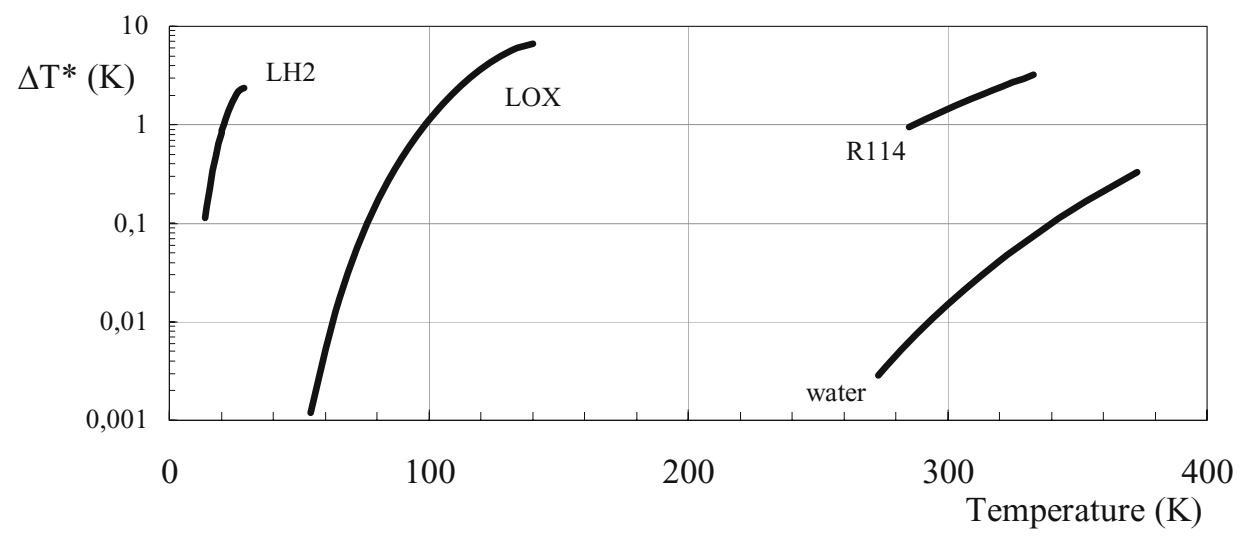

Figure 19. Values of $\Delta T^{*}$ for liquid hydrogen, liquid oxygen, refrigerant 114 and water at different temperatures

By combining equations (5.2), (5.6) and (5.7), the drop in vapor pressure for the growing bubble is then:

$$
\Delta p_{v} \cong \rho_{\ell} \Sigma \dot{R} \sqrt{t}
$$

where parameter $\Sigma$, originally introduced by Brennen, is defined by:

$$
\Sigma=\frac{\left(\rho_{v} L\right)^{2}}{\rho_{\ell}{ }^{2} c_{p_{\ell}} T_{\infty} \sqrt{\alpha_{\ell}}}
$$

Like $\Delta T^{*}$, the $\Sigma$ parameter, which has units of $\mathrm{m} / \mathrm{s}^{3 / 2}$, increases significantly with thermal effects. It can then be considered as another indicator of the magnitude of the thermodynamic effect. Table 1 gives typical values for cold and hot water.

\begin{tabular}{|l|l|l|}
\hline WATER & $20^{\circ} \mathrm{C}$ & $100^{\circ} \mathrm{C}$ \\
\hline$\Delta T^{*}(\mathrm{~K})$ & 0.0102 & 0.324 \\
\hline$\Sigma(\mathrm{m} / \mathrm{s} 3 / 2)$ & 3.89 & 2944 \\
\hline
\end{tabular}

Table 1. Values of $\Delta T^{*}$ and $\Sigma$ for water at $20^{\circ} \mathrm{C}$ and $100^{\circ} \mathrm{C}$

To come back to the growth of a cavitation bubble in case thermal effects are not negligible, consider the Rayleigh equation (2.3):

$$
R \ddot{R}+\frac{3}{2} \dot{R}^{2}=\frac{p_{v}\left(T_{c}\right)-p_{\infty}}{\rho_{\ell}}
$$

In this equation, the vapor pressure which holds for the pressure inside the bubble is taken at temperature $T_{c}$. Introducing the vapor pressure at the liquid bulk temperature $T_{\infty}$, we obtain:

$$
\left(R \ddot{R}+\frac{3}{2} \dot{R}^{2}\right)+\frac{\Delta p_{v}}{\rho_{\ell}}=\frac{p_{v}\left(T_{\infty}\right)-p_{\infty}}{\rho_{\ell}}
$$


This equation is similar to the usual Rayleigh equation except concerning the second term on the left hand side which is new and accounts for thermal effects. Using equation (5.8), this term is transformed as follows:

$$
\left(R \ddot{R}+\frac{3}{2} \dot{R}^{2}\right)+\Sigma \dot{R} \sqrt{t} \cong \frac{p_{v}\left(T_{\infty}\right)-p_{\infty}}{\rho_{\ell}}
$$

The thermal term, which is initially zero, takes increasing importance with time. Hence, thermal effects remain negligible as long as:

$$
\Sigma \dot{R} \sqrt{t}<<\frac{p_{v}\left(T_{\infty}\right)-p_{\infty}}{\rho_{\ell}}
$$

Since the bubble growth rate $\dot{R}$ is of the order of $\sqrt{\left(p_{v}\left(T_{\infty}\right)-p_{\infty}\right)} / \rho_{\ell}$, the previous condition becomes:

$$
t<\frac{p_{v}\left(T_{\infty}\right)-p_{\infty}}{\rho_{\ell} \Sigma^{2}}
$$

In the practical case of a cavitating flow of characteristic velocity $V$ and characteristic length scale $L$ as that around a blade of chord length $L$, the typical time $t$ available for bubble growth is the transit time $L / V$. It has to be compared to the characteristic time $\left(p_{v}\left(T_{\infty}\right)-p_{\infty}\right) /\left(\rho_{\ell} \Sigma^{2}\right)$ to estimate whether thermal effects significantly affect cavitation or not. In this equation, $p_{\infty}$ is the pressure applied to the bubble and responsible for its growth whose typical value is the minimum pressure on the foil. For a given fluid and given cavitating conditions, it is then quite easy from equation (5.14) to evaluate the importance of the thermodynamic effect. In particular, it is clear that the larger $\Sigma$, the more important the thermodynamic effect.

\section{Supercavitation}

Supercavity flows as the one shown in figure 5 are characterized by a long cavity whose length may be much larger than that of the cavitator which generates it. The closure region of a supercavity takes place in the liquid bulk, usually far downstream the cavitator. The smaller the cavitation number, the longer the cavity.

We will consider here the case of an axisymmetric supercavity flow past a cavitator as shown in figure 20. For long cavities, the slender body approximation can be applied. It consists in assuming that the body and its cavity bring only a slight perturbation to the basic flow and then that the axial flow velocity is everywhere close to the velocity at infinity $V_{\infty}$. Although $V_{\infty}$ is assumed constant, the present approach applies to unsteady supercavities when unsteadiness is due to a time dependent pressure at infinity $p_{\infty}$ or a time dependent pressure inside the cavity $p_{c}$.

The latter occurs especially in ventilated flows when the cavity is generated by blowing non condensable gas (e.g. air) in the wake of the cavitator. Ventilated flows are similar to natural cavity flows, in spite of some differences connected to the non condensable nature of the injected gas in comparison to vapor, and are then relevant of the same type of approach. A particular feature of ventilated flows is the possibility for the cavity to have a pulsating behavior connected to a periodic release of air in the wake. If so, the cavity length oscillates periodically and the cavity 
pressure $p_{c}$ too. Pulsating ventilated supercavities is an example of unsteady supercavity flows which can be modeled by the present approach.

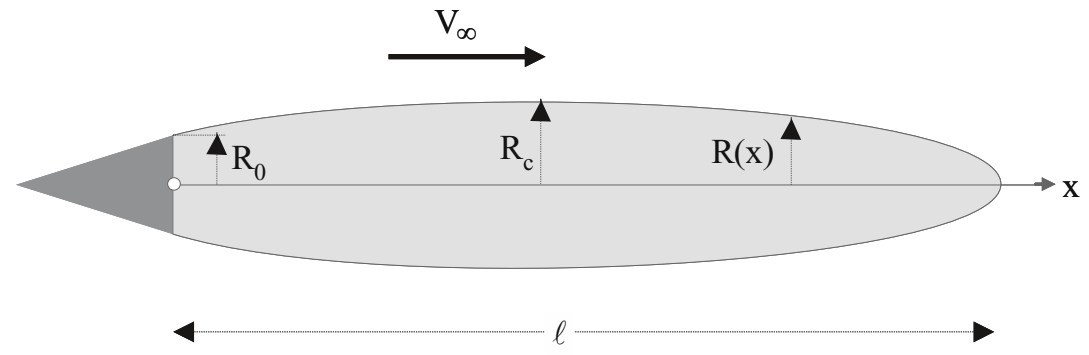

Figure 20. Axisymmetric supercavity flow

Consider a cross section area of the cavity at station $\mathrm{x}$. The corresponding radius and section are respectively $R$ and $S=\pi R^{2}$. Both depend on the axial position $x$ and in addition on time $t$ in the unsteady case. It can be shown (cf. e.g. Franc \& Michel, (2004)) that the evolution of the cross section area $S$ is given by the following equation:

$$
\frac{d^{2} S}{d t^{2}}=-\frac{2 \pi}{\mu} \frac{p_{\infty}-p_{c}(t)}{\rho}
$$

This equation is derived from Euler equation using the mass conservation equation together with the slender body approximation. $d / d t$ is the usual transport derivative given by:

$$
\frac{d}{d t}=\frac{\partial}{\partial t}+V_{\infty} \frac{\partial}{\partial x}
$$

The $\mu$ parameter takes into account the inertia of the liquid surrounding any section of cavity and is equivalent to an added mass coefficient. It is generally assumed constant and can be related to the cavitation number $\sigma$. In particular, its asymptotic behavior when $\sigma$ approaches zero i.e. for long supercavities is the following (see Franc \& Michel, (2004)):

$$
2 \mu \cong \ln \frac{1}{\sigma}
$$

By using $S=\pi R^{2}$, equation (6.1) becomes:

$$
\rho\left[R \ddot{R}+\dot{R}^{2}\right]=\frac{1}{\mu}\left(p_{c}-p_{\infty}\right)
$$

where $\dot{R}$ and $\ddot{R}$ stand respectively for $d R / d t$ and $d^{2} R / d t^{2}$. Equation (6.1) is then very similar to the Rayleigh equation except for the coefficient of $\dot{R}^{2}$ which is $3 / 2$ in the original Rayleigh equation for the spherical bubble and which is here 1 in axisymmetric configurations. This similarity gives a basis for the physical interpretation of equation (6.1).

Consider a given cross section of the axisymmetric cavity. The use of the transport derivative $d / d t$ suggests to follow it as it is advected by the main flow at velocity $V_{\infty}$. During its movement, the radius of this cut of "cylindrical" bubble changes according to equation (6.4). Like for a spherical bubble, the driving term for the change in radius is the pressure difference between the pressure at infinity and the internal pressure. As shown by equation (6.4), the dynamics of any cross section of the cavity depends only upon this pressure difference and, in particular, it does 
not depend upon the neighboring cross sections. This result is known as the Logvinovich independence principle of cavity expansion.

It can be considered that, at any time, a new section of cavity is shed by the cavitator basis. It is advected downstream at velocity $V_{\infty}$ whereas its radius evolves according to the pressure difference $p_{\infty}-p_{c}$. The instantaneous shape of the whole cavity is obtained by stacking all sections side by side.

The initial conditions to be added for the resolution of partial differential equation (6.4) are related to the instant when the considered section of the cavity separates from the cavitator basis. Its initial radius $R$ is then the radius of the cavitator basis whereas the initial value of $\dot{R}$ is related to the slope of the cavitator trailing edge. It must be such that continuity of slope is achieved at any time between the cavitator and the cavity at detachment, resulting then in a smooth detachment.

The resolution of partial differential equation (6.1) allows the computation of the shape of the supercavity and its time evolution if the flow is unsteady. As an example, consider the simple case of a steady supercavity flow for which $d / d t$ reduces to $V_{\infty} d / d x$. Equation (6.1) becomes then:

$$
\frac{d^{2} R^{2}}{d x^{2}}=-\frac{\sigma}{\mu}
$$

The solution to this equation is obviously:

$$
\left(\frac{R}{R_{0}}\right)^{2}=\frac{x}{R_{0}}\left[2 \dot{R}_{0}-\frac{\sigma}{2 \mu} \frac{x}{R_{0}}\right]+1
$$

where $R_{0}$ is the radius of the cavitator basis at $x=0$ and $\dot{R}_{0}$ its slope $d R / d x$ at $x=0$. The origin $x=0$ is chosen at the cavitator basis. For the asymptotic case considered here of a long supercavity at small cavitation number, the radius of the cavity is much larger than the radius $R_{0}$ of the cavitator, so that the last term on the right hand side can be neglected and the shape of the cavity is then given approximately by:

$$
\left(\frac{R}{R_{0}}\right)^{2} \cong \frac{x}{R_{0}}\left[2 \dot{R}_{0}-\frac{\sigma}{2 \mu} \frac{x}{R_{0}}\right]
$$

This equation shows that the cavity can be approximated by an ellipsoid. The cavity length $\ell$, which corresponds to the downstream point where $R$ equals 0 , is then given by:

$$
\frac{\ell}{R_{0}} \cong \frac{4 \mu}{\sigma} \dot{R}_{0}
$$

The maximum radius $R_{c}$ of the cavity is obtained at $x=\ell / 2$ and is:

$$
\frac{R_{c}}{R_{0}} \cong \sqrt{\frac{2 \mu}{\sigma}} \dot{R}_{0}
$$

so that cavity slenderness $\delta=2 R_{c} / \ell$ is:

$$
\delta=\sqrt{\frac{\sigma}{2 \mu}}
$$


When the cavitation number approaches 0 , the $\mu$ parameter tends to infinity according to asymptotic behavior 6.3 . The length and maximum radius of the cavity both tend to infinity but the cavity slenderness tends to zero according to the following asymptotic behavior:

$$
\delta \cong \sqrt{\frac{\sigma}{\ln (1 / \sigma)}}
$$

Previous equations (6.8) and (6.9) are more often written using the drag coefficient $C_{D}$ instead of $\dot{R}_{0}$. The drag can be computed from a global momentum balance. It is given by (see e.g. Franc \& Michel, (2004)):

$$
D \cong\left(p_{\infty}-p_{c}\right) \pi R_{c}^{2}
$$

so that the drag coefficient is:

$$
C_{D}=\frac{D}{\frac{1}{2} \rho V_{\infty}^{2} \pi R_{0}^{2}} \cong \sigma\left(\frac{R_{c}}{R_{0}}\right)^{2} \cong 2 \mu \dot{R}_{0}^{2}
$$

Replacing $\dot{R}_{0}$ by $C_{D}$ in equations (6.8) and (6.9), the following equations for the length and radius of the axisymmetric supercavity are obtained:

$$
\left\{\begin{array}{l}
\frac{\ell}{R_{0}} \cong \frac{2}{\sigma} \sqrt{2 \mu C_{D}} \cong \frac{2}{\sigma} \sqrt{C_{D} \ln \frac{1}{\sigma}} \\
\frac{R_{C}}{R_{0}} \cong \sqrt{\frac{C_{D}}{\sigma}}
\end{array}\right.
$$

These equations are well known asymptotic formulae which were originally derived by Garabedian in 1956 (see Franc \& Michel, (2004)) on the basis of a rather complicated mathematical approach. The method presented here and based on equation (6.1) is quite simple. However, to be fully predictive, the method requires to model the $\mu$ parameter which is unknown a priori. This is done via equation (6.3) for instance which gives the asymptotic behavior of $\mu$ when $\sigma$ approaches zero. In practice, equation (6.3) was obtained by adjusting the value of $\mu$ in order to get the correct value of the cavity slenderness as given by the Garabedian solution. It is then quite normal that equations (6.14) be in agreement with the Garabedian model. Other procedures of adjustment of the $\mu$ parameter could however be used, by comparison with tests for instance.

In conclusion, it appears that axisymmetric supercavity flows can be modelled by an equation very similar to the Rayleigh equation, providing some simplifying assumptions. It is also the case for 2D supercavity flows (see Pellone et al. (2004)). One of the advantages of such a method is to provide a rather simple basis for the modeling of supercavity flows, especially in unsteady cases which are not so easy to handle. The adjustment of $\mu$ obtained from steady considerations is generally kept for unsteady supercavity flows. In particular, this method proved to be quite efficient for the analysis of pulsating ventilated cavities (see Franc \& Michel, (2004)).

\section{Cavitation erosion}

It is well known that cavitation can lead to erosion. Figure 21 presents a typical example of a component of a volumetric pump eroded by cavitation. For low exposure times, the damage re- 
mains superficial. It is characterized by small isolated pits, whose diameter ranges typically from a few microns to a fraction of millimeter and whose depth is of the order of a few percents of their diameter, and even less. Each of them is caused by the collapse of a distinct vapor structure. This preliminary stage, without significant mass loss, is the incubation period. The material surface undergoes mainly plastic deformation.

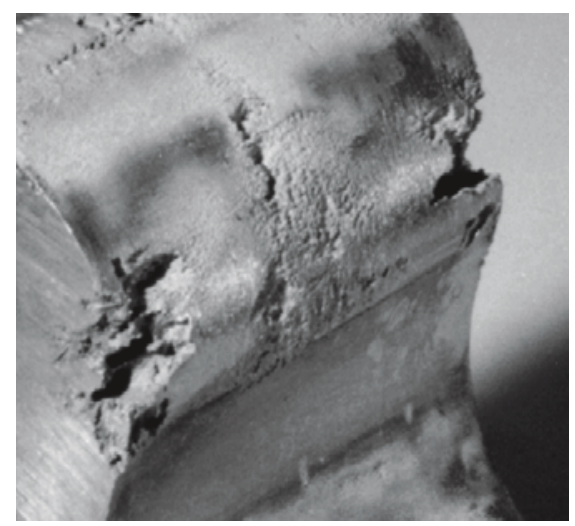

Figure 21. A typical example of cavitation erosion

When the exposure time increases, overlapping becomes more and more important. The wall is then submitted to successive collapses whose number increases with exposure time. Repetitive loading occurs, leading to fatigue followed by rupture and subsequent material removal. During this advanced stage of erosion, the damage rate is often characterized by the mean depth of penetration rate (MDPR) i.e. the thickness of material which is removed per unit exposure time.

The aggressiveness of a cavitating flow depends largely upon flow velocity. There is a threshold velocity below which no damage occurs whatever may be the exposure time. In the case of water and stainless steel $316 \mathrm{~L}$, this threshold normally lies around 15 to $20 \mathrm{~m} / \mathrm{s}$. Above, the erosion rate increases very rapidly with velocity. The influence of flow velocity on MDPR is often modeled by a power law with a large exponent, typically between 4 and 9 .

As for the evolution of erosion with the development of cavitation, it is generally observed that damage, which is of course zero under non cavitating conditions, first increases when the cavitation number decreases and then decreases for developed cavitation.

The objective of this section is to point out a few basic trends in cavitation erosion from the consideration of the only Rayleigh-Plesset equation. It is shown that trends of practical interest can be obtained concerning for instance scaling rules in cavitation erosion or the influence of cavitation types on the erosive potential.

\subsection{Bubble collapse and cavitation erosion}

Firstly, the Rayleigh equation will be used to explain why a bubble, when collapsing in the vicinity of a wall, can actually damage it. The existence of damage implies that the wall has been loaded with a stress larger than its yield strength so that plastic deformation actually occurred. 
Consider for instance the case of stainless steel $316 \mathrm{~L}$ whose yield strength is about $400 \mathrm{MPa}$. The occurrence of pits proves that the collapse of vapor structures generates impact loads larger than $400 \mathrm{MPa}$ or, in other words, pressure pulses larger than 4000 bar. The point is then to understand how a bubble can generate such a high pressure when collapsing.

The answer partly lies in the distribution of pressure within the fluid during collapse. Consider a pure vapor bubble which is collapsing because of a pressure $p_{\infty}$ applied far from the bubble greater than the vapor pressure $p_{v}$ which prevails inside the bubble (cf. section 3.3). If surface tension effects are neglected, pressure on the liquid side at the bubble interface is also $p_{v}$. Pressure distribution must then increase from $p_{v}$ to $p_{\infty}$ when moving away from the bubble interface.

It is remarkable that, if at the very beginning of the collapse, pressure smoothly increases from $p_{v}$ to $p_{\infty}$, it is no longer the case as soon as the bubble radius becomes smaller than a given fraction of its initial radius, namely $63 \%$. From that time, pressure distribution exhibits a maximum very close to the bubble wall as shown in figure 22 . This maximum increases all along the collapse phase. Hence, the collapsing bubble gives rise to a kind of pressure wave which propagates inward and steepens during collapse. It must be noticed that this pressure wave is not due to compressibility, which is ignored in the present model, but results only of inertia forces.

The computation of the pressure field inside the liquid during collapse can be found in most textbooks on cavitation (see for example Franc and Michel, (2004)). At any time t, if the bubble radius is $\mathrm{R}(\mathrm{t})$, the maximum pressure $p_{\max }$ is given by the approximate following non dimensional equation:

$$
\Pi=\frac{p_{\max }-p_{\infty}}{p_{\infty}-p_{v}} \cong 0.157\left(\frac{R_{0}}{R}\right)^{3}
$$

where, as usually $R_{0}$ is the initial bubble radius. Furthermore, the analytical computation shows that this maximum takes place close to the bubble wall at a radial distance $r$ from the bubble center of the order of:

$$
r \cong 1.59 R
$$

Both equations (7.1) and (7.2) are asymptotic formulas valid for small enough values of $R / R_{0}$ i.e. near the end of the collapse.

As a consequence, a high pressure is generated in the liquid, close to the bubble. Such a high pressure is due to the always increasing velocity of the bubble wall and the focusing effect of the spherical collapse. At the end of the collapse, the interface velocity tends to infinity and the maximum pressure too as shown by equation (7.1). As an example, for a vapor bubble collapsing under pressure at infinity $p_{\infty}=1 \mathrm{bar}$, the maximum pressure reaches almost $1 \mathrm{GPa}$ when the bubble radius is divided by $50\left(R_{0} / R=50\right)$. This value is quite comparable to yield strength and even ultimate strength of usual materials, so that damage can actually be expected. 

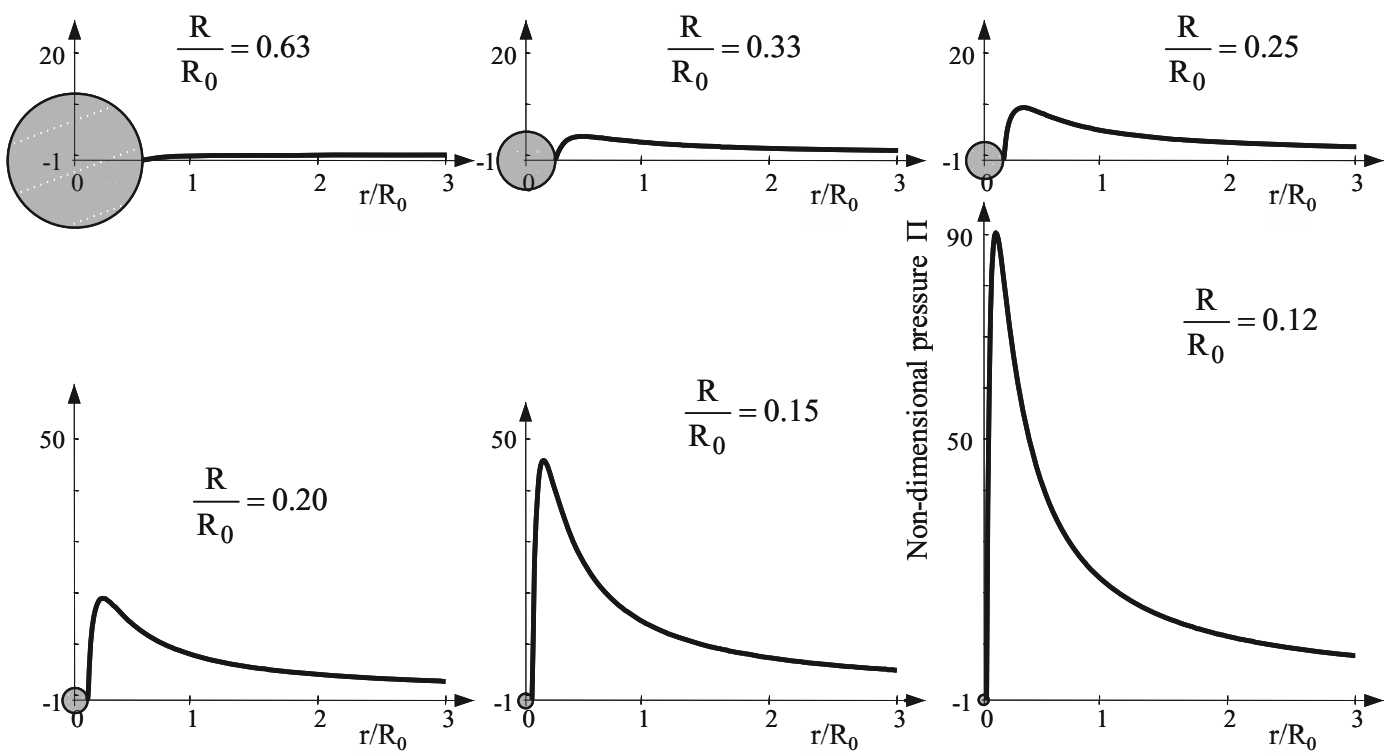

Figure 22. Pressure distribution in the liquid at different times during bubble collapse

As already mentioned in section 3.3, the infinite values given by the present model are not realistic physically and other phenomena (as compressibility and gas content), negligible at the beginning of the collapse, become predominant at the end. Therefore, the actual physics is somewhat more complicated than that given by this simple model.

As an example, the presence of non condensable gas inside the bubble results in a high internal pressure at the end of the collapse which generally forces the bubble to rebound. Computations as well as experiments have shown that an expanding shock wave of high amplitude is generated at the instant of rebound. The impact of this shock wave on the wall is a possible cause of damage.

Another plausible mechanism is the development of a microjet during collapse. As a matter of fact, when a bubble collapses near a wall, spherical symmetry is obviously broken by the wall and the bubble does not remain spherical. A liquid microjet develops towards the wall, goes through the bubble and strikes the wall at high velocity. The resulting impact pressure can also be a reason for damage.

In addition, collective effects, when a cloud of bubbles is collapsing for instance, may also play a role in the damaging process.

Although there is still some controversy on the detailed physical mechanism of cavitation erosion, it is unquestionable that a high pressure is generated during the collapse of a bubble as shown by the Rayleigh equation. Note that the damage potential depends greatly on the distance of the bubble to the wall. The closer to the wall, the higher the impact pressure. 


\subsection{Scaling law for the aggressiveness of a single bubble}

According to the simple previous model (cf. equation (7.1)), the peak of pressure generated by the collapse of a bubble is proportional to the driving pressure $p_{\infty}-p_{v}$ responsible for the collapse and to the ratio $\left(R_{0} / R\right)^{3}$ :

$$
p_{\max } \approx\left(p_{\infty}-p_{v}\right)\left(\frac{R_{0}}{R}\right)^{3}
$$

Besides, equation (3.4) shows that the velocity of the bubble interface during collapse is given by:

$$
\dot{R} \approx \sqrt{\frac{p_{\infty}-p_{v}}{\rho}\left(\frac{R_{0}}{R}\right)^{3}}
$$

so that, according to this model, the pressure peak scales like:

$$
p_{\text {max }} \approx \rho \dot{R}^{2}
$$

as it could be expected from simple dimensional analysis.

If we refer to the microjet model mentioned in the previous section, assuming that the damage is produced by a liquid jet impacting the wall at high velocity still denoted $\dot{R}$, the impact pressure can be estimated using the classic water hammer formula:

$$
p_{\text {max }} \approx \rho c \dot{R}
$$

where $\rho c$ is the acoustic impedance of the liquid. Note that this equation is valid in the case of a perfectly rigid wall and that a correction has to be applied in case the acoustic impedance of the liquid is not negligible with respect to that of the material.

Although some differences exist between the two models in particular with respect to the exponent of $\dot{R}$, a general conclusion is that the aggressiveness of the collapsing bubble depends primarily upon the interface velocity $\dot{R}$. The point is now to know how the interface velocity scales with the velocity and length scale of the mean flow.

Consider the cavitating flow around a foil as already analyzed in section 4.2 and let us investigate the effect of a change either in length scale (typically the chord length $L$ ) or in flow velocity $V$ on the velocity $\dot{R}$ of the bubble interface. As explained in section 4.2 , if the bubble radius is made non dimensional using the chord length $L$ of the foil and if time derivatives are replaced by space derivatives, the Rayleigh equation takes the following non dimensional form (cf. equation (4.10)):

$$
\bar{R} \frac{d^{2} \bar{R}}{d \bar{x}^{2}}+\frac{3}{2}\left(\frac{d \bar{R}}{d \bar{x}}\right)^{2}=-\frac{1}{2}\left(C_{p}+\sigma\right)
$$

For geometrically similar flows - i.e. for the same distribution of pressure coefficient $C_{p}-$ and for equal cavitation numbers $\sigma$-i.e. for equal developments of cavitation -, it was already observed that the evolution of the non dimensional bubble radius $\bar{R}(\bar{x})$ along the foil is unique.

Furthermore, from the non dimensional procedure presented in section 4.2, it can easily be shown that the dimensional value of the velocity of the bubble interface is: 


$$
\dot{R}=V \frac{d \bar{R}}{d \bar{x}}
$$

This proves that, for similar flows, $\dot{R}$ is proportional to mean flow velocity. In other words, the dynamics of a bubble traveling on the foil is scaled as the mean flow velocity.

Another consequence of equation (7.8) is that the bubble interface velocity is independent of the length scale which does not appear in equation (7.8). If chord length is doubled for instance, bubble radius is expected to be doubled too. Since the flow velocity is assumed to be the same, the transit time is also doubled, so that finally the bubble interface velocity is unchanged.

The important conclusion of this analysis is that the velocity of the bubble interface depends essentially upon mean flow velocity and that the dependence should be linear. Such a result has to be considered as a first order trend and deviations can be expected due to secondary phenomena as interactions between bubbles, viscous effects... This result is used in next section to derive scaling laws for the aggressiveness of a cavitating flow.

\subsection{Scaling laws for flow aggressiveness}

In the previous section, it has been shown that the aggressiveness of a collapsing bubble is proportional to flow velocity. However, as mentioned in the introduction, cavitation damage increases much more rapidly with flow velocity. An analysis of scaling laws for the aggressiveness of a cavitating flow allows to better understand this influence.

A cavitating flow is characterized by a large population of bubbles of various characteristics. If we consider a given surface element of a wall exposed to cavitation, it is quite common to describe flow aggressiveness at this location by a pressure pulse height spectrum (PPHS) $\dot{n}(\Delta p)$. It represents the density $\dot{n}$ per unit time and unit surface area of pressure pulses whose amplitude is larger than $\Delta p$.

Considering still similar cavitating flows around blades or hydrofoils as an example, it is expected that PPHS is a function of flow velocity $V$ and chord length $L$ which is chosen as characteristic length scale as previously. In addition, PPHS also depends upon the fluid. A priori, several fluid properties may influence flow aggressiveness as density, viscosity, surface tension... Referring to the simplified approach developed in section 7.2 where inertia is considered as predominant, a unique physical property for the liquid will be considered here as relevant for cavitation erosion. It is either the fluid density $\rho$ if we refer to equation (7.5) or the acoustic impedance $\rho c$ if we refer to equation (7.6).

We will then write either:

$$
\dot{n}=\operatorname{function}(\Delta p, V, L, \rho)
$$

or:

$$
\dot{n}=\operatorname{function}(\Delta p, V, L, \rho c)
$$

Basic dimensional analysis leads to the following non dimensional relations:

$$
\frac{\dot{n} L^{3}}{V}=\varphi_{1}\left(\frac{\Delta p}{\rho V^{2}}\right)
$$


or:

$$
\frac{\dot{n} L^{3}}{V}=\varphi_{2}\left(\frac{\Delta p}{\rho c V}\right)
$$

where $\varphi_{1}$ (respectively $\varphi_{2}$ ) is a universal function for the considered flow configuration independent of length sale, velocity and fluid properties, provided that geometrical and cavitation scaling are both fulfilled.

To simplify, we will abandon the fluid dependence and consider a unique fluid. Only the influence of length scale and velocity on PPHS will then be examined. The approach could however be extended to account for the effect of other factors on cavitation erosion as a change of material.

To be more general, equations (7.11) and (7.12) are written as follows:

$$
\frac{\dot{n} L^{3}}{V}=\varphi\left(\frac{\Delta p}{V^{\alpha}}\right)
$$

where exponent $\alpha$ is a parameter which ranges a priori between 1 and 2. A common form for function $\varphi$ is a power law with a negative exponent $-\beta$. A typical order of magnitude for $\beta$ is 2 to 4 (see e.g. Franc and Michel (2004), Kato et al. (1996)). However, data on $\beta$ are only few and the $\beta$ value might significantly depend upon flow configuration. With this choice, PPHS is written as follows:

$$
\dot{n}=A \frac{V^{\alpha \beta+1}}{L^{3}} \frac{1}{\Delta p^{\beta}}
$$

It is then possible to estimate pitting rate on a given material. The approach is very basic. The material is characterized by its yield strength $\sigma_{Y}$ and it is assumed that a pit is formed if the pulse height $\Delta p$ is larger than $\sigma_{Y}$ and that only elastic behavior occurs otherwise. Pitting rate i.e. the density of pits per unit surface area and unit exposure time is then:

$$
\dot{n}_{\text {pits }}=A \frac{V^{\alpha \beta+1}}{L^{3}} \frac{1}{\sigma_{Y}^{\beta}}
$$

Hence, it appears that pitting rate increases as the power $\alpha \beta+1$ of flow velocity. Assuming typical values for $\alpha$ and $\beta$ of 1.5 and 3, we have $\alpha \beta+1 \cong 5.5$. Hence, this approach shows that flow aggressiveness increases with a relatively high power of flow velocity, as shown by experiments.

Two reasons explain this strong influence of flow velocity. Firstly, the aggressiveness of a single bubble increases with flow velocity, as already noticed. Secondly, for a cavitating flow, an increase in flow velocity results in an increase in the production rate of vapor structures. This can be seen on equations (7.11) or (7.12). If we consider vapor structures which follow scaling law (7.5) or (7.6), then, for a constant value of either $\Delta p / \rho V^{2}$ or $\Delta p / \rho c V$, equations (7.11) and (7.12) show that:

$$
\frac{\left(\dot{n} L^{2}\right) L}{V}=\text { Constant }
$$


This can be considered as a Strouhal scaling law since $\dot{n} L^{2}$ is actually a frequency representing the rate of collapse of vapor structures on similar surface areas per unit time. Hence, the collapsing rate increases proportionally to flow velocity. The increase of bubble production rate with flow velocity combined with the increase of the aggressiveness of single bubbles with flow velocity results in a relatively strong increase of the overall aggressiveness of a cavitating flow.

Previous conclusions apply to similar cavitating flows presenting, in particular, the same type of cavitation. Besides, qualitative trends can also be obtained as for the effect of cavitation types on flow aggressiveness. Two main factors have a great influence: the maximum bubble size prior to collapse and the adverse pressure gradient which forces the bubble to collapse. From a qualitative viewpoint, an increase in bubble size has the same effect as an increase in pressure gradient. Both tend to increase the erosive potential.

From an erosion viewpoint, it is then preferable to reduce as much as possible the adverse pressure gradient in order to lengthen bubble lifetime and then minimize the violence of collapse. On the other hand, a small adverse pressure gradient has a destabilizing effect on cavity closure. The length of the cavitating zone and consequently the whole cavitating flow is prone to fluctuations and unsteadiness. In practice, the control of adverse pressure gradient is mainly a matter of design and it may be necessary to find a compromise between erosive potential and flow stability.

Besides pressure gradient, maximum bubble size prior to collapse is another essential parameter for the evaluation of erosive potential. Consider the case of traveling bubble cavitation. For a given flow configuration, bubble maximum size depends greatly upon nuclei density. If it is small, bubbles are rather big. If it is large, bubble growth is limited by neighboring bubbles. This results in smaller bubbles and smaller erosive potential, too. For traveling bubble cavitation, it can then be expected that flow aggressiveness will depend upon nuclei density since it affects maximum bubble size.

As for attached cavitation, its aggressiveness depends upon the type of cavity. An attached closed and stable cavity usually produces small bubbles which are concentrated in the closure region of the cavity. Cavitation damage is then focused at cavity closure where bubbles collapse. This is the case for instance of a thin leading edge cavity attached to a blade at small angle of attack.

An increase in angle of attack leads to an increase in cavity thickness. The cavity becomes more open and sheds more bubbles in its wake. Since the bubble production rate increases, the erosive potential is expected to increase. Moreover, damage generally extends on a larger zone, upstream and downstream of mean cavity closure.

Unsteadiness can significantly enhance the erosive potential of a cavitating flow. It is more especially the case of cloud cavitation which can be quite aggressive from an erosion viewpoint. This is due first to the high density of bubbles contained in a cavitation cloud and also to collective effects during the collapse of a bubble cluster which may enhance the intensity of each elementary collapse. 


\section{Vortex cavitation}

Although the standard Rayleigh-Plesset equation applies to spherical bubbles only, the dynamics of other types of cavitating structures can often be predicted by very similar equations.

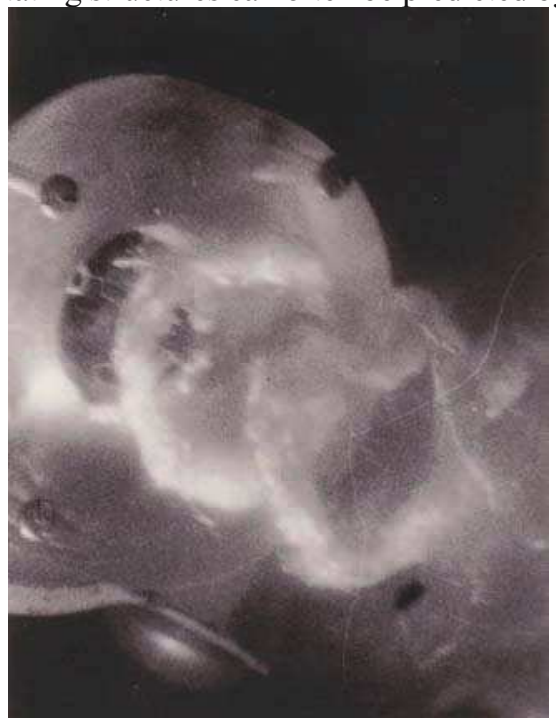

Figure 23. Cavitating vortex rings issuing from a StratoJet ${ }^{\circledR}$ self-resonating cavitating jet. Resonance is achieved through a feedback mechanism between the shear layer at the nozzle lip and the upstream organ pipe tube leading to the orifice. The frequency of emission of the rings is the same as the organ pipe frequency (Courtesy of Dr. Georges Chahine from Dynaflow, Inc.)

It is in particular the case of toroidal cavitating vortices or "ring bubbles" as those periodically produced by a cavitating submerged round jet (figure 23). Chahine and Genoux (1983) have shown that the time evolution of the radius $R(t)$ of a cross section of the ring bubble follows an equation analogous to the Rayleigh-Plesset equation:

$$
\rho\left[R \ddot{R}+\dot{R}^{2}\right] \ln \frac{8 A}{R}-\frac{1}{2} \rho \dot{R}^{2}=\left[p_{v}-p_{\infty}(t)\right]+\frac{1}{2} \rho\left[\frac{\Gamma}{2 \pi R}\right]^{2}+p_{g 0}\left(\frac{R_{0}}{R}\right)^{2 k}-\frac{S}{R}
$$

On the right hand side, we can identify the same terms as in the original Rayleigh-Plesset equation i.e. the driving pressure term together with the non condensable gas and surface tension terms with however slight changes due to differences in geometry. In addition to these conventional terms, an additional one appears which depends upon circulation $\Gamma$ of the ring bubble. Indeed, such a ring bubble is a toroidal vortex which is provided with a vorticity characterized by the $\Gamma$ parameter. Physically, circulation tends to resist the collapse in a similar way as non condensable gas. Another parameter which is specific to ring bubbles and which appears on the left hand side of equation (8.1) is the radius $A$ of the ring (see figure 23). Nevertheless, as for the spherical bubble, equation (8.1) allows the analysis of various characteristics of a bubble ring as its collapse time or its stability. 
It can also be shown that the dynamics of an axisymmetric cavitating vortex is governed by a Rayleigh-Plesset like equation. Further information on cavitating vortices can be found in Chahine \& Genoux (1983) and Franc \& Michel (2004).

\section{Cavitation modeling}

As a first example of the capabilities of the Rayleigh-Plesset equation to model real cavitating flows, let us consider the case of a partial cavity attached to the suction side of a foil as shown in Figure 3. This type of cavity is obviously far from being spherical. However, at least in a preliminary step, it can be approached using the Rayleigh-Plesset equation as follows.

Consider the original fully-wetted flow corresponding to the cavitating case presented in Figure 3. Its characteristics - and particularly the pressure field - are assumed to be known from classical computational techniques. Consider an isolated spherical (or more commonly hemispherical as observed in Figure 6) bubble whose center moves along the wall. This bubble is assumed to be initially a cavitation nucleus i.e. a microbubble of only a few microns in diameter. For small enough values of the cavitation number, this bubble, which is carried by the flow, will first grow in the low pressure region generated by the foil, become a macroscopic cavitation bubble and then collapse downstream where the pressure recovers. To some extent, the envelope of the hemispherical bubble as it moves along the foil gives a rough outline of the original attached cavity. Then, the development of cavitation can be analyzed, at least qualitatively, on the basis of the simple Rayleigh-Plesset equation.

Such a simplified model has obviously many serious limitations. In particular, it ignores any feedback of cavitation on the original fully wetted flow pressure field, which can be considered as valid only near cavitation inception. More realistic, but also more complicated models, can be used according to the accuracy required for prediction. Many of them are still based upon the Rayleigh-Plesset equation as it is the case for the bubble two-phase flow model derived by Kubota et al. (1992) whose principle is presented below.

The framework of the modeling is the standard homogeneous model which provides the simplest technique for analyzing two-phase flows. The liquid/vapor mixture is treated as a pseudofluid which obeys the usual equations of single-phase flow, i.e. continuity:

$$
\frac{\partial \rho}{\partial t}+\operatorname{div}(\rho \vec{V})=0
$$

and momentum balance or Navier-Stokes equation :

$$
\rho\left[\frac{\partial \vec{V}}{\partial t}+(\vec{V} \cdot g r \vec{a} d) \vec{V}\right]=-\operatorname{gra} d p+\mu\left[\vec{\Delta} \vec{V}+\frac{1}{3} \operatorname{gra} d(\operatorname{div} \vec{V})\right]
$$

In the previous equations, $\vec{V}$ is the velocity of this pseudofluid and $\rho$ its density. Slip between liquid and vapor (bubbles actually) is ignored, so that a unique velocity $\vec{V}$ is considered for the two-phase mixture.

A major feature of the model is the large range of variation of density. Consider the case of water at ambient temperature as a typical example. Density can vary between $\rho_{\ell}=1000 \mathrm{~kg} / \mathrm{m}^{3}$ 
in regions occupied by the liquid alone and $\rho_{v}=0.017 \mathrm{~kg} / \mathrm{m}^{3}$ in pure vapor cavities. From a numerical viewpoint, the large density ratio of the order of 60000 between liquid and vapor is a real difficulty which generally requires special computational techniques in order to ensure the stability of the numerical scheme.

To be solved, equations (9.1) and (9.2) must be completed by a constitutive equation for this pseudofluid. Some models use an explicit equation. It is the case for instance of the barotropic model which assumes that density is a continuous function of pressure with a drop from the pure liquid density to the pure vapor density at vapor pressure.

The bubble two-phase flow model does not assume any explicit constitutive equation for the two phase mixture. The liquid is supposed to carry nuclei with a bubble number density $\mathrm{n}$. They grow in the low pressure region before collapsing where the pressure recovers. Any cavitating zone is then considered as a kind of bubble cluster.

The density of the two-phase mixture is:

$$
\rho=\alpha \rho_{v}+(1-\alpha) \rho_{\ell}
$$

where $\alpha$ is the void fraction. A similar averaging procedure can be used for viscosity. $\alpha$ is computed on the basis of the bubble number density $n$ and bubble radius $R$ by:

$$
\alpha=n \cdot\left(\frac{4}{3} \pi R^{3}\right)
$$

As for the radius, it is computed using the Rayleigh equation:

$$
R \frac{d^{2} R}{d t^{2}}+\frac{3}{2}\left(\frac{d R}{d t}\right)^{2}=\frac{p_{v}-p}{\rho_{\ell}}
$$

where $d / d t$ is the usual transport derivative and $p$ the local pressure.

The model, made of the five equations (9.1) to (9.5), allows in principle the computation of the five unknowns $\rho, \vec{V}, p, \alpha$ and $R$ at any time and in any cell of the computational domain.

Several variants have been developed. The original version of Kubota et al. (1992) is based upon an improved version of the Rayleigh-Plesset equation (9.5) which takes into account interactions between bubbles of a cluster, limiting bubble growth in comparison to the case of an isolated bubble. A simplified version has also been used by considering instead of equation (9.5) the asymptotic growth rate 3.3:

$$
\dot{R} \cong \sqrt{\frac{2}{3} \frac{p_{v}-p}{\rho}}
$$

During the resorption or collapse phase for which $p>p_{v}$, a similar equation can be used:

$$
\dot{R} \cong-\sqrt{\frac{2}{3} \frac{p-p_{v}}{\rho}}
$$

Although such a model appears to be appropriate more especially to cloud cavitation, it has been applied successfully to other types of cavitation including even leading edge cavitation. In practice, it may be necessary to limit artificially the void fraction to 1 depending on the computed configuration and model used. 
The bubble number density $n$ is a free parameter in the model. It is often assumed constant all over the computational domain. A typical value is $10^{6}$ nuclei $/ \mathrm{m}^{3}$ i.e. $1 \mathrm{nucleus} / \mathrm{cm}^{3}$. The initial bubble radius is also a free parameter. Both may have an influence on the cavitating flow structure as noticed by Kubota et al. (1992). More sophisticated models can be imagined using a variable bubble number density. They aim at modeling the effects of coalescence and fragmentation of bubbles which is still a difficult question.

A key parameter of such a model is the vapor production rate $\dot{m}_{v}$ which measures the mass of vapor that cavitation locally produces (or resorbs) per unit volume and unit time. It appears as a source term in the continuity equation for the vapor phase:

$$
\frac{\partial\left(\alpha \rho_{v}\right)}{\partial t}+\operatorname{div}\left(\alpha \rho_{v} \vec{V}\right)=\dot{m}_{v}
$$

and with the opposite sign in that for the liquid phase:

$$
\frac{\partial\left[(1-\alpha) \rho_{\ell}\right]}{\partial t}+\operatorname{div}\left[(1-\alpha) \rho_{\ell} \vec{V}\right]=-\dot{m}_{v}
$$

Summation of equations (9.8) and (9.9) results in the continuity equation (9.1) for the liquid mixture. By developing equation (9.1) with definition (9.3) of the density for the mixture, a transport equation for void fraction can be obtained (liquid and vapor densities are assumed constant):

$$
\frac{\partial \alpha}{\partial t}+\vec{V} \cdot \operatorname{gra} d \alpha=\frac{\rho}{\rho_{\ell}-\rho_{v}} \operatorname{div} \vec{V}
$$

Comparison of equations (9.8) and (9.10) shows that $\operatorname{div} \vec{V}$ is directly related to the vapor production rate:

$$
\dot{m}_{v}=\frac{\rho_{\ell} \rho_{v}}{\rho_{\ell}-\rho_{v}} \operatorname{div} \vec{V}
$$

In conclusion, to close the system made of continuity and Navier-Stokes equations for the two-phase mixture, it is necessary to introduce a cavitation model which specifies the vapor production rate $\dot{m}_{v}$. The bubble two-phase flow model is one of them. The choice of the cavitation model is crucial for the results of the simulation.

The present section gave only an overview of the principle of simulation of real cavitating flows using the Rayleigh equation. To be effective, such a mathematical model must be associated to an advanced numerical method capable of accounting for large variations in density.

\section{Conclusion}

Although restrictive assumptions are associated to the Rayleigh-Plesset equation which, strictly speaking, applies to a single spherical bubble in an infinite liquid medium at rest at infinity, various aspects of real cavitating flows can be analyzed, at least qualitatively but sometimes also quantitatively, using this equation. A simple way to obtain general conclusions is to write it in non dimensional form. Depending upon the practical situation and the objectives which are looked for, there are several ways to make it non dimensional, each of them being characterized by a different choice of the relevant time scales and/or length scales. 
The use of the bubble radius as characteristic length scale allows the evaluation of the relative importance of pressure, viscosity and surface tension effects and to find out the one which controls bubble evolution. The use of a length scale which characterizes the cavitating flow itself - as the chord length of a blade if, for example, the cavitating flow around a blade is concerned allows the derivation of scaling laws. As an example, this kind of approach makes it possible to develop guidelines for the analysis of the influence of flow velocity on the erosive potential of a cavitating flow.

The Rayleigh-Plesset equation also applies to other types of cavities, different from the spherical bubble, as cavitating vortices, providing minor adjustments. Moreover, any cross section of a supercavity can also been modeled by a Rayleigh-Plesset type equation, in the framework of the Logvinovich independence principle. Hence, there is quite a variety of cavities whose dynamics can be approached by an equation analogous to the Rayleigh-Plesset equation.

Finally, this equation is widely used for the numerical modeling of complex real cavitating flows. A usual procedure consists in assuming that the liquid carries cavitation nuclei whose growth is controlled by the Rayleigh-Plesset equation. This is a simple way to account for the production of vapor in a cavitating flow by pressure reduction. The corresponding source term is introduced in the continuity equation for the vapor phase which is solved numerically together with the Navier-Stokes equation for the liquid/vapor mixture.

\section{Bibliography}

Arndt R.E.A. (2002) Cavitation in vertical flows. Annu. Rev. Fluid Mech. 34 143-175

Blake J.R. and Gibson D.C. (1987) Cavitation bubbles near boundaries. Annu. Rev. Fluid Mech. 19 99-128

Brennen C.E. (1995) Cavitation and bubble dynamics. Oxford University Press

Chahine G.L. and Genoux P.F. (1983) Collapse of a cavitating vortex ring. J. Fluids Eng. 105 400-405

Escaler X., Farhat M., Avellan F. and Egusquiza E. (2003) Cavitation erosion tests on a 2D hydrofoil using surface-mounted obstacles. Wear 254 441-449

Franc J.-P. and Michel J.-M. (2004) Fundamentals of Cavitation. Kluwer

Fruman D.H., Reboud J.L. and Stutz B. (1999) Estimation of the thermal effects in cavitation of thermosensible liquids. Int. J. Heat and Mass Transfer 42 3195-3204

Fujikawa S. and Akamatsu T. (1980) Effects of non-equilibrium condensation of vapour on the pressure wave produced by the collapse of a bubble in a liquid. J. Fluid Mech. 97 part 3 481-512

Garabedian P.R. (1956) Calculation of axially symmetric cavities and jets. Pac. J. Math. 6 611-684

Gopalan S. and Katz J. (2000) Flow structure and modeling issues in the closure region of attached cavitation. Physics of Fluids 12 No.4 895-911

Kato H., Konno A., Maeda M. and Yamaguchi H. (1996) Possibility of quantitative prediction of cavitation erosion without model test. J. Fluids Eng. 118 582-588.

Knapp R.T., Daily J.W. and Hammitt F.G. (1970) Cavitation. McGraw-Hill 
Kubota A., Kato H. and Yamaguchi H. (1992) A new modeling of cavitating flows: a numerical study of unsteady cavitation on a hydrofoil section. J. Fluid Mech. 240 59-96.

Lecoffre Y. (1995) Cavitation erosion, hydrodynamics scaling laws, practical method of long term damage prediction. Proc. Int. Symp. on Cavitation Deauville (France) 249-256

Leighton T.G. (1994) The acoustic bubble. Academic Press

Lindau O. and Lauterborn W. (2003) Cinematographic observation of the collapse and rebound of a laserproduced cavitation bubble near a wall. J. Fluid Mech. 479 327-348

Logvinovich G.V. (1969) Hydrodynamics of free surface flows (in Russian). Nauvoka Dunka Ed. Kiev

Pellone C., Franc J.-P. and Perrin M. (2004) Modelling of unsteady 2D cavity flows using the Logvinovich independence principle. C.R. Mécanique 332 827-833.

Plesset M.S. (1949) The dynamics of cavitation bubbles. J. Appl. Mech. 16277 sq.

Prosperetti A. (2004) Bubbles. Physics of Fluids 16 No.6 1852-1865

Rayleigh (Lord) (1917) The pressure developed in a liquid during the collapse of a spherical cavity. Phil. Mag. 3494 sq.

Serebryakov V.V. (1972) The annular model for calculation of axisymmetric cavity flows (in Russian). Hydromechanics Nauvoka Dunka Ed. Kiev 27 25-29

Tomita Y., Robinson P.B., Tong R.P. and Blake J.R. (2002) Growth and collapse of cavitation bubbles near a curved rigid boundary. J. Fluid Mech. 466 259-283

Trevena D.H. (1987) Cavitation and tension in liquids. Adam Hilger

Young F.R. (1989) Cavitation. McGraw-Hill 